\title{
The bright spiky emission of pulsar B0656+14
}

\author{
P. Weltevrede ${ }^{1}$, G. A. E. Wright ${ }^{2,1}$, B. W. Stappers ${ }^{3,1}$, and J. M. Rankin ${ }^{4,1}$ \\ 1 Astronomical Institute "Anton Pannekoek", University of Amsterdam, Kruislaan 403, 1098 SJ Amsterdam, The Netherlands \\ e-mail: wltvrede@science.uva.nl \\ 2 Astronomy Centre, University of Sussex, Falmer, BN1 9QJ, UK \\ e-mail: G.Wright@sussex.ac.uk \\ 3 Stichting ASTRON, Postbus 2, 7990 AA Dwingeloo, The Netherlands \\ e-mail: stappers@astron.nl \\ 4 Physics Department, 405 Cook Physical Science building, University of Vermont, Burlington 05405, USA \\ e-mail: Joanna. Rankin@uvm. edu
}

Received 9 May 2006 / Accepted 13 July 2006

\section{ABSTRACT}

Context. We present a detailed study of the single radio pulses of PSR B0656+14, a pulsar also known to be a strong pulsed source of high-energy emission.

Aims. Despite the extensive studies at high-energy wavelengths, there is little or no published work on its single-pulse behaviour in the radio band. In this report we rectify this omission.

Methods. Radio observations using the Westerbork Synthesis Radio Telescope at $1380 \mathrm{MHz}$ and the Arecibo Observatory at 327 and $1525 \mathrm{MHz}$ are used to investigate the single-pulse behavior of PSR B0656+14. A comparison is made with the phenomena of giant pulses and giant micropulses.

Results. We have found that the shape of the pulse profile of PSR B0656+14 requiresan unusually long timescale to achieve stability (over 25000 pulses at $327 \mathrm{MHz}$ ). This instability is caused by very bright and narrow pulses with widths and luminosities comparable to those observed for the RRATs. Many pulses are bright enough to qualify as "giant pulses", but are broader than those usually meant by this term. At $327 \mathrm{MHz}$ the brightest pulse was about 116 times brighter than the average pulse. Although the most powerful pulses peak near the centre of the profile, occasional sudden strong pulses are also found on the extreme leading edge of the profile. One of them has a peak flux of about 2000 times the average flux at that pulse longitude. No "break" in the pulse-energy distributions is observed, but nevertheless there is evidence of two separate populations of pulses: bright pulses have a narrow "spiky" appearance consisting of short quasi-periodic bursts of emission with microstructure, in contrast to the underlying weaker broad pulses. Furthermore, the spiky pulses tend to appear in clusters which arise and dissipate over about 10 periods. We demonstrate that the spiky emission builds a narrow and peaked profile, whereas the weak emission produces a broad hump, which is largely responsible for the shoulders in the total emission profiles at both high and low frequencies.

Key words. stars: pulsars: individual: PSR B0656+14 - stars: pulsars: general - radiation mechanisms: non-thermal

\section{Introduction}

PSR B0656+14, at a spin-down age of $111000 \mathrm{yr}$, is one of three nearby pulsars in the middle-age range in which pulsed high-energy emission has been detected. These are commonly known as "The Three Musketeers" (Becker \& Trümper 1997), the other two being Geminga and PSR B1055-52. Middle-aged pulsars (roughly defined to be those whose spin-down ages range from $50000 \mathrm{yr}$ to $300000 \mathrm{yr}$ ) are of interest to neutron star theorists because they allow the detection of high-energy thermal radiation from the star's surface, and hence can provide tests for models of surface cooling and atmosphere composition. In more energetic young pulsars (the Crab) the pulsed non-thermal emission is dominant, and completely masks the thermal emission at all wavelengths. In cool, older pulsars blackbody surface temperatures are expected to have fallen below the detection threshold, with the likely exception of their heated polar caps (the polar cap of one such older pulsar, B0943+10, having recently been detected in soft X-rays (Zhang et al. 2005).

However pulsars such as B0656+14 are also of interest because they provide tests between competing emission models for the highest energy components. Their hard X-ray emission can variously be interpreted as a product of outergaps located in the outer magnetosphere (Cheng \& Zhang 1999), or as originating closer to the surface magnetic poles (Harding \& Muslimov 1998). Fundamental to these discussions are the determination of the geometric location of the various high-energy peaks, and their relation to the radio emission peak, which is generally taken to define the polar cap of the pulsar's dipole axis. In the case of PSR B0656+14, a major clue is that observations from optical to hard X-ray all show one or both of the two peaks always at the same two phase longitudes (De Luca et al. 2005; Kern et al. 2003; Pavlov et al. 2002; Shibanov et al. 2005; Zavlin et al. 1996), while the radio peak falls almost, but not quite, halfway between these peaks.

PSR B0656+14 was included in a recent extensive survey of subpulse modulation in pulsars in the northern sky (Weltevrede et al. 2006a). In the single pulses analysed for this purpose the unusual nature of this pulsar's emission was very evident, especially the "spiky" nature of the subpulses. But what was most striking was that the pulsar occasionally exhibited exceptionally powerful and longitudinally narrow subpulses reminiscent of "giant" pulses, hitherto reported for only a handful of pulsars, including the Crab pulsar (Staelin \& Reifenstein 1968) and 
mostly young or millisecond pulsars (e.g. Soglasnov et al. 2004). More recently, giant pulses have been discovered in two old pulsars (Kuzmin \& Ershov 2004). If their presence could be confirmed in PSR B0656+14, this would demonstrate that the phenomenon might also be found in pulsars of intermediate age and hence at any stage of a pulsar's lifetime.

We therefore set out to explore the full nature of PSR B0656+14's pulse behaviour in the radio band. PSR B0656+14 has a period $(0.385 \mathrm{~s})$ not greatly below the average for all pulsars and at radio frequencies exhibits an apparently unremarkable single-peak integrated profile. Yet at any wavelength the integrated profile of a pulsar conceals as much information as it yields, and this pulsar has proved to be no exception. It is remarkable that, despite the extensive studies at high-energy wavelengths, there is little or no published work on its single-pulse behaviour in the radio band. In this report we rectify this omission, and, in addition to confirming the presence of extremely bright pulses across the profile, demonstrate that the pulsar's radio emission is far from that typical of older better-known pulsars.

While this work was being prepared the discovery of RRATs (Rotating RAdio Transients) was announced. These are sources which emit single powerful pulses, separated by long intervals, and were identified as isolated pulsars with periods of between 0.4 and $7 \mathrm{~s}$. However, their relation to the known pulsar population was unclear. The intermittent giant pulses we have detected in PSR B0656+14 led us to argue in a separate paper that this pulsar, were it not so near, could itself have appeared as an RRAT (Weltevrede et al. 2006b). This paper is complementary to that work, showing that this pulsar's bright pulses are narrow and "spiky", occur at a wide range of central longitudes and can be differentiated from a weaker but steadier underlying emission. A third paper is planned in which we will present polarization data and attempt to link the radio emission - in a single geometric structure - to the high-energy peaks.

The details of the radio observations are described in the next section. In Sect. 3 the pulse profile is discussed, in Sect. 4 the properties of the single pulses and in Sect. 5 we will show that the emission can be decomposed into "spiky" and "weak" emission. In Sect. 6 our results are discussed and summarized.

\section{Radio observations}

\subsection{Introduction}

Initially, a relatively short sequence of about two thousand pulses of PSR B0656+14 were collected with the Westerbork Synthesis Radio Telescope (WSRT) to analyze its subpulse modulation properties as part of a large survey for subpulse modulation at $21 \mathrm{~cm}$ (Weltevrede et al. 2006a). It was found that the intensity of the single pulses is modulated with frequencies larger than about 10 pulse periods and the modulation index $m$ was found to be high, indicating that the intensity variation of the single pulses is unusual. The single pulses of this pulsar would have not been further investigated had not one of them triggered our interest: a single exceptionally bright pulse on the leading edge far from the centre of the pulse profile, which quickly led us to realise that the emission of the pulsar was far from normal. This pulsar was then observed by the WSRT for five more hours (of which two proved highly suitable because of fortunate interstellar scintillation conditions). Archival Arecibo Observatory (AO) data was also studied as well as a follow-up observation. The details of the observations used in this paper can be found in
Table 1. The details of the observations used in this paper. Here REF is the reference key used in this paper, MJD is the modified Julian date of the observation, $v$ is the central frequency of the observation, $\Delta v$ the bandwidth, $\tau_{\text {samp }}$ the sampling time and $N$ the number of recorded pulses.

\begin{tabular}{c|rrrll}
\hline \hline REF & MJD & $\begin{array}{c}v \\
(\mathrm{MHz})\end{array}$ & $\begin{array}{c}\Delta v \\
(\mathrm{MHz})\end{array}$ & $\begin{array}{c}\tau_{\text {samp }} \\
(\mathrm{ms})\end{array}$ & $N$ \\
\hline AO-P1 & 52840 & 327 & 25 & 0.5125 & 24765 \\
AO-P2 & 53490 & 327 & 25 & 0.650 & 16888 \\
AO-L & 52854 & 1525 & 100 & 0.5125 & 15589 \\
WSRT-L & 53437 & 1380 & 80 & 0.2048 & 18586 \\
\hline
\end{tabular}

Table 1 (including a reference key, which we use throughout this paper).

\subsection{Cleaning of the data}

The WSRT data was of good quality without any Radio Frequency Interference (RFI) to be removed, but the AO data did suffer from some RFI. Pulses contaminated by RFI could relatively easily be identified by calculating the root-mean-square (rms) of the off-pulse noise. From all AO observations the pulses with the highest off-pulse rms (about $1 \%$ of the data) were identified and excluded from further analysis. The AO-P2 observation is, although shorter, of better quality than the older AO-P1 observation.

The AO-L observation was affected by lightning, which caused spikes in the data. To avoid confusing lightning with bright pulses from the pulsar, we had to identify and remove the artificial spikes. This was done by checking the data for the presence of non-dispersed spikes in the frequency band. Using this method we were able to identify 46 spikes from the data which were removed by replacing them by samples with the running baseline values, after which the data was de-dispersed.

\section{The shape and stability of the pulse profile}

\subsection{Shape of the pulse profiles}

The average profiles of two of the AO observations are shown in Fig. 1 and the top and middle profile are the pulse profiles at two different frequencies. The shape of the pulse profile of PSR B0656+14 is unusual for radio pulsars, especially at high frequencies, where the profile is practically an isosceles triangle. In fact, it proved impossible to fit this profile with any Gaussian or Lorentzian form.

The profile at $1525 \mathrm{MHz}$ is composed of an almost symmetric central peak with a weak shoulder (from pulse longitude $\sim 190^{\circ}$ to $\sim 210^{\circ}$ ) on the trailing side of the profile. At $327 \mathrm{MHz}$ the pulse profile shape becomes more complex. Although the profile is dominated by the central peak (the leading part of the profile up to pulse longitude $\sim 190^{\circ}$ ), the shoulder (visible up to pulse longitude $\sim 200^{\circ}$ ) is more prominent and a second shoulder appears (up to pulse longitude $\sim 220^{\circ}$ ). The central peak seems to show some structure at $327 \mathrm{MHz}$ (there is an excess at pulse longitude $\sim 182^{\circ}$ ), which is also visible in the AO-P2 observation. Note that the profiles are wide with a full-width-half-maximum $(F W H M)$ of $18.9^{\circ}$ and $14.0^{\circ}$ at $327 \mathrm{MHz}$ and $1525 \mathrm{MHz}$ respectively and a $10 \%$ width to $39.4^{\circ}$ and $28.8^{\circ}$ at $327 \mathrm{MHz}$ and $1525 \mathrm{MHz}$ respectively. 


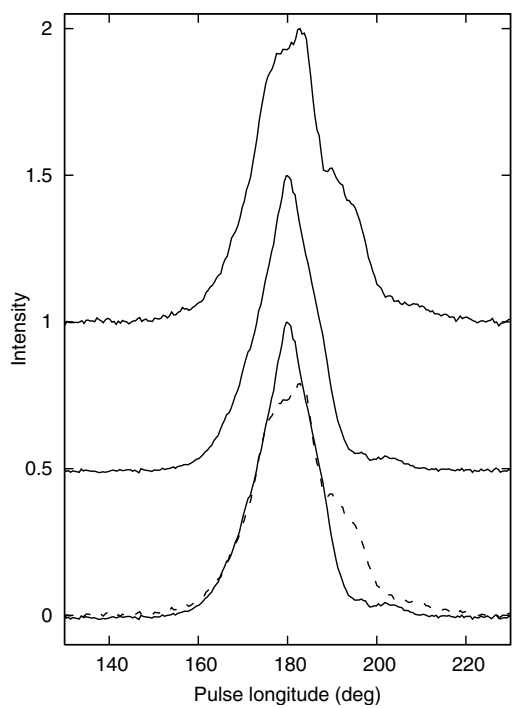

Fig. 1. The top and middle profiles (solid line) are the normalized pulse profiles of the $327-\mathrm{MHz}$ AO-P1 and $1525-\mathrm{MHz}$ AO-L observation respectively which are plotted with a vertical offset of 0.5 and 1 . The profiles are aligned such that the central peak falls at pulse longitude $180^{\circ}$ and the two profiles are plotted overlaid at the bottom of the figure. The intensity of the $327-\mathrm{MHz} \mathrm{AO}-\mathrm{P} 1$ is scaled such that the central peaks fit each other.

\subsection{Alignment of the pulse profiles}

It is important to keep in mind that the observations are not time aligned, so the profiles of each observation have an arbitrary pulse longitude offset. At the bottom of Fig. 1 we have overlaid a 327 and 1525-MHz profile, keeping the centroid of the central peak at a fixed pulse longitude. The pulse profiles are scaled in intensity such that the central component overlaps. Although the relative shift and scaling are subjective, the fact that the profiles fit so accurately is surprising and shows that the central component of the two profiles maintains an invariant width over frequencies more than a factor four apart.

This alignment suggests that the profile almost exclusively evolves with frequency on the trailing side. The pulse profile becomes broader at lower frequencies as expected from radius-tofrequency mapping (RFM, Cordes 1978). However it is doubtful whether this is actually the origin of the broadening. One could argue from the bottom profiles of Fig. 1 that there is no RFM at all in the central peak and that the broadening of the pulse profile is only due to the appearance of more emission components.

\subsection{Stability of the pulse profile}

For most pulsars one can obtain a stable pulse profile by averaging a few to a few hundred pulses, and the profiles of younger pulsars tend to stabilize more quickly (Helfand et al. 1975; Rathnasree \& Rankin 1995), so our observations of up to 25000 pulses could have been expected to be long enough. However PSR B0656+14 proved to be far from typical, and the stability of its profile turns out to be an important issue in determining the "true" shape of its pulse profile.

To test whether our data sets are long enough to get a stable pulse profile, we split the data into two halves and computed their average profiles. The overlaid profiles in the left panels of Fig. 2 show the average profiles of the first and second halves of two observations. As one can see, the profiles of the two halves of the observation are similar, but with significant differences.
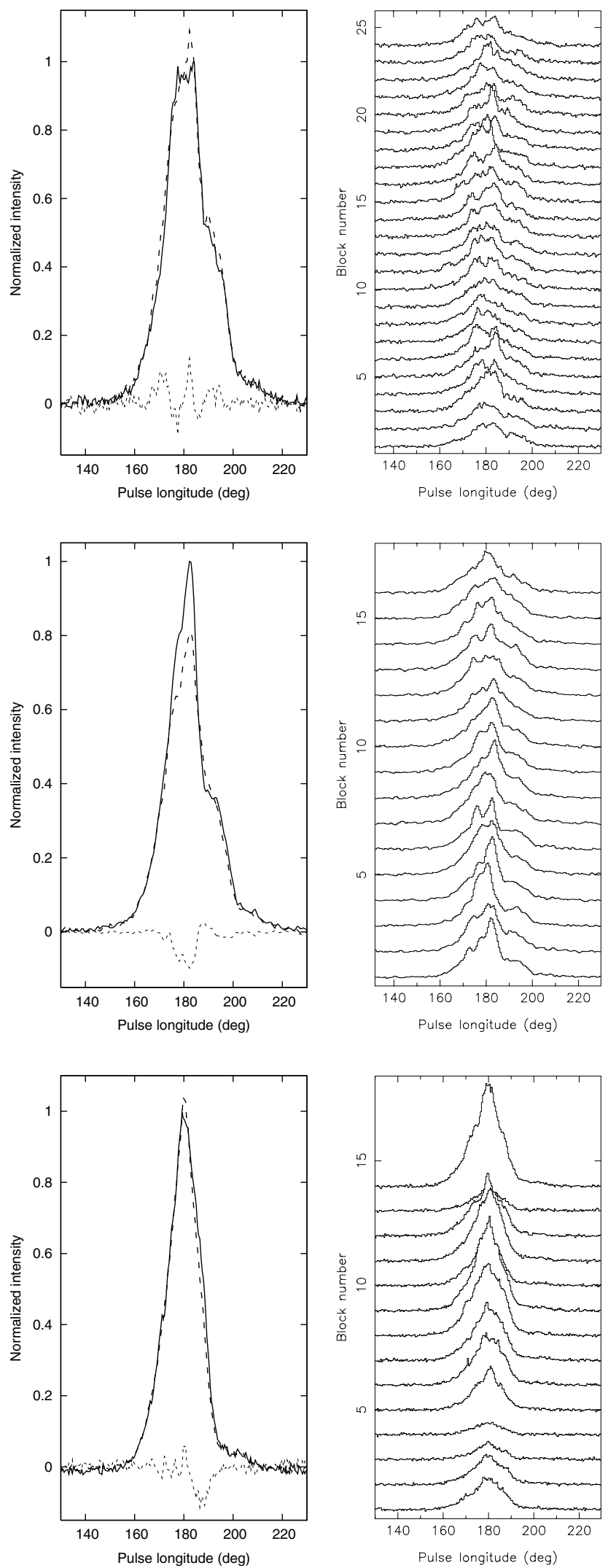

Fig. 2. From top to bottom these panels show the $327-\mathrm{MHz}$ AO-P1, AO-P2 and 1525-MHz AO-L observations. The overlaid profiles of the left panels are the average-pulse profiles of the first (solid line) and second halves (dashed line) of the observations and the dotted lines are their differences. Only the two overlaid profiles in the bottom left panel are scaled in intensity to fit each other. The profiles of the right panels are the pulse profiles obtained by averaging successive blocks of one thousand pulses each. 
This is especially the case on the trailing side of the $1525-\mathrm{MHz}$ pulse profile and in the central peak of the $327-\mathrm{MHz}$ profiles.

To further illustrate the time dependence of the pulse profile, the profiles of successive blocks of one thousand pulses were calculated (right panels of Fig. 2). Unlike the 327-MHz profiles (top and middle right panels), the $1525-\mathrm{MHz}$ profiles (bottom right panel) have different intensities because of interstellar scintillation. This suggests that at $327 \mathrm{MHz}$ the scintillation bandwidth is much smaller than the observing bandwidth. In Sect. 3.4 we will argue that this is indeed the case. Note that apart from the intensity changes due to scintillation the shape of the profile is much more stable at high frequencies compared with low frequencies.

As one can also see in Fig. 2, the profile shape changes are very significant on timescales of one thousand pulses, especially at $327 \mathrm{MHz}$. A much longer observation would be required to find out if there exists a time scale for the pulse profile to stabilize. The profiles in the top left panel of Fig. 2 are not scaled in intensity to match and are unaffected by scintillation. This means that the radio power output of the pulsar at $327 \mathrm{MHz}$ is very stable, although the profile shape is not.

In the middle left panel of Fig. 2, the pulse profiles of the first and second halves of the AO-P2 observation are shown overlaid. Unlike the AO-P1 observation (top left Fig. 2), the intensity has significantly changed during the observation. Note that the intensity of the central peak has changed in height, while the intensities of the two shoulders remains the same. The middle right panel of Fig. 2 confirms that the pulse profile is changing gradually around pulse 11000 (block 11) from a more peaked to a much broader shape. So not only is the profile highly unstable on short timescales, it can also evolve gradually on a timescale of hours.

It must be noted that a non-equatorial mounted telescope with cross-coupling errors can introduce a gradual time-evolution in the shape of (highly linear polarized) pulse profiles as a function of parallactic angle. However, the observations are calibrated for polarization and the parallactic-angle rotation is taken into effect ${ }^{1}$. Moreover, the particular type of cross-coupling which occurs in the AO instrument is well studied and has the property of preserving both total and polarized power. Therefore, systematic errors due to polarization calibration uncertainties are probably not responsible for the observed effects.

\subsection{Scintillation properties}

The dynamic spectrum of the 1380-MHz WSRT-L observation is shown in Fig. 3. One can see that the scintles (the dark patches) have a characteristic timescale (the scintillation timescale) of the order of a thousand seconds. The horizontal stripes in the dynamic spectrum are because the $80-\mathrm{MHz}$ bandwidth of the observation is divided into eight $10-\mathrm{MHz}$ bands. At the edges of the bands there is no useful data because the bandpass rolls off just inside the full-width of $10 \mathrm{MHz}$. The scintillation bandwidth (the characteristic frequency scale of the scintles) is clearly less than the total bandwidth of the observation $(80 \mathrm{MHz})$. In the thin screen approximation, the scintillation bandwidth is proportional to $v^{4}$ (Scheuer 1968), close to the observed scaling (e.g. Bhat et al. 2004). Using this scaling one expects a scintillation bandwidth smaller than $0.3 \mathrm{MHz}$ at $327 \mathrm{MHz}$. This is much smaller than the observing bandwidth of the AO observations at that

1 PSR B0656+14 if far enough away from the Arecibo zenith to fully accommodate parallactic-angle rotation.

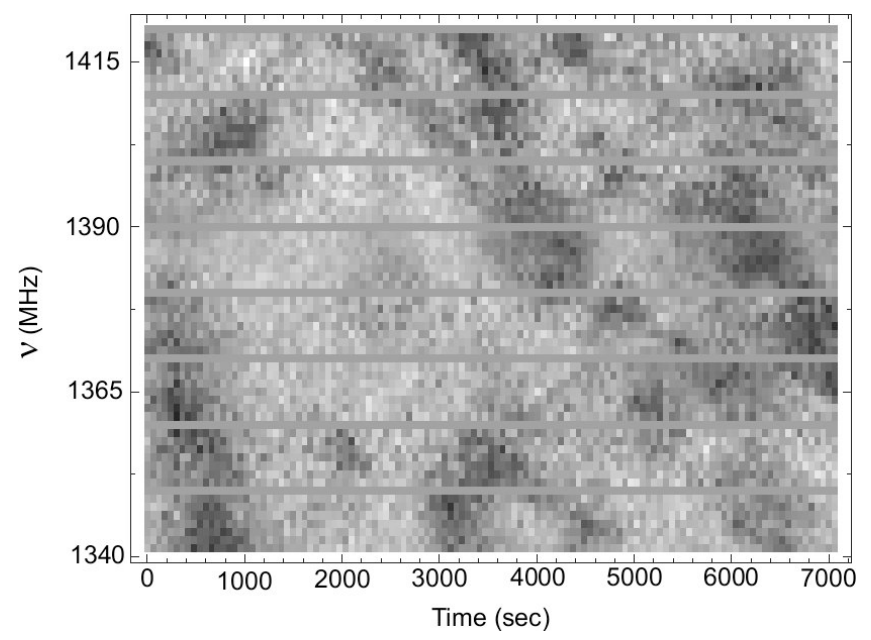

Fig. 3. The dynamic spectrum of the 1380-MHz WSRT-L observation. Here the measured signal to noise of the pulsar signal is plotted in grayscale as a function of both time and frequency. The horizontal stripes in the dynamic spectrum show where the eight frequency-bands roll off.

frequency $(25 \mathrm{MHz})$. Therefore the effect of the individual scintles will be averaged out at $327 \mathrm{MHz}$, causing intensity fluctuations due to scintillation to be invisible in the top and middle panels of Fig. 2. In fact, the scintillation bandwidth at this frequency is so small that it is comparable, or even smaller than, the the bandwidth of the individual frequency channels $(0.098 \mathrm{MHz})$. This means that it is not possible to measure it directly from our observations, which is confirmed in the middle panel of Fig. 8.

\section{The radio bursts of PSR B0656+14}

A typical pulse sequence of this pulsar at $327 \mathrm{MHz}$ is shown in Fig. 4. The plotted pulse-longitude range is the whole range within which the pulsar is found to emit (compare with Fig. 2). One can see that the frequent outbursts of radio emission are much narrower than the width of the pulse profile. The emission also has burst-like behaviour in the sense that the radio outbursts tend to cluster in groups of a few pulse periods. Furthermore, this clustering sometimes seems to be weakly modulated with a quasi-periodicity of about 20 pulse periods (see for instance the bursts around pulse numbers 55, 75, 95, 115 and 135). Apart from these bursts there are many pulses (and large fractions of the pulse window) that contain no signal above the noise level.

We will use the term spiky to refer to these bursts of radio emission. In this section we investigate the energy distributions of this pulsar, leading to comparisons with the phenomena of giant pulses and giant micropulses.

\subsection{Correction for scintillation}

To compare the bright pulses of PSR B0656+14 with the giant pulse phenomenon, we have calculated the pulse-energy distributions. Because the dispersion measure (DM) is quite low for this pulsar, scintillation can be severe. From Figs. 2 and 3 it is clear that we have to be careful in interpreting the energy distributions of the high frequency observations. Without correction, the pulse energies will be over- and underestimated when interstellar scintillation makes the pulsar signal respectively stronger and weaker. To correct for this broadening of the pulse-energy distribution, the pulse energies of the two high frequency observations are compared with the running average of the pulse 


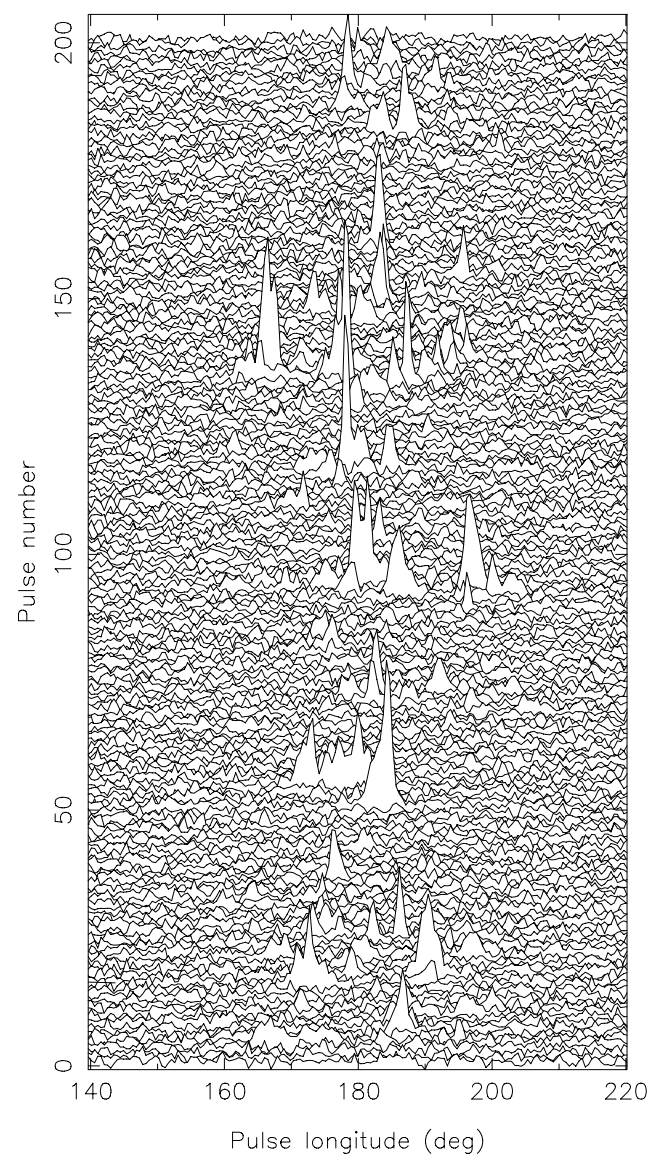

Fig. 4. A typical sequence of successive pulses of the 327-MHz AO-P1 observation.

energies (which we will denote by $\langle E\rangle$ ), instead of simply with the average-pulse energy of the whole observation.

We used a running average of 600 pulses. This timescale is chosen such that the running average smoothly follows the scintillation (which has a timescale of the order of one thousand seconds). This timescale is also sufficiently large compared with the timescale of the pulse-intensity modulation ( $\sim 20$ pulse periods, as we will show in Sect. 5.6). To prevent the running average from being influenced too much by very bright pulses, we do not include pulses above $6\langle E\rangle$ (the effect of this correction turns out to be negligible). The correction for scintillation will broaden the off-pulse energy distribution. This is because when scintillation makes the pulsar weaker, the noise rms (expressed in terms of the running-average pulse energy $\langle E\rangle$ ) becomes larger. To improve the signal-to-noise $(S / N)$ ratio, a threshold for the running average is used. If $\langle E\rangle$ drops below this threshold, the pulses are not used for the scintillation-corrected pulse-energy distribution.

\subsection{Giant pulses?}

The scintillation-corrected high-frequency pulse-energy distributions are shown in Fig. 5, together with that for the 327-MHz AO-P2 observation which did not require correction. The pulseenergy distributions extend over an extremely wide energy range. In the AO-P2 observation the brightest pulse is about $116\langle E\rangle$ (outside the plotted energy range). This is well above the "working definition of giant pulses" threshold of $10\langle E\rangle$ (e.g. Cairns 2004). In the 327-MHz AO-P1 and AO-P2 observation respectively $0.4 \%$ and $0.2 \%$ of the pulses are above this threshold. In the $1525-\mathrm{MHz} \mathrm{AO}-\mathrm{L}$ and in the $1380-\mathrm{MHz}$ WSRT observation this percentage is an order of magnitude less $(0.04 \%$ and $0.01 \%$ ).

PSR B0656+14 therefore emits giant pulses in the sense that it emits single pulses with energies above $10\langle E\rangle$. Another characteristic of giant pulses is that they are very narrow with timescales down to nano-seconds (e.g. Soglasnov et al. 2004; Hankins et al. 2003). Also the giant pulses of most pulsars are emitted in a small pulse-longitude range compared with the pulse profile. In Fig. 6 one can see an example of a bright pulse from PSR B0656+14 which shows they have clear structure and are much broader than the "classical" giant pulses. In order to determine whether the bright pulses are associated with certain locations in the pulse profile, we have added the single pulses within certain pulse-energy ranges (left panels of Fig. 7). It is clear that the brightest pulses with $E>10\langle E\rangle$ are not strictly confined to a small pulse-longitude range. Nonetheless they are limited to the leading and central regions, i.e. the shoulders are clearly associated with the weak pulses. Especially at $327 \mathrm{MHz}$ there is a remarkably sharp boundary between the central peak and the shoulder for the occurrence of the bright pulses. The profile of the summed brightest pulses of the 327-MHz AO-P1 observation appears to have a separate component on the leading side of the pulse profile (at pulse longitude $165^{\circ}$ ). However only 15 pulses contribute to this component, which is furthermore not visible in the AO-P2 observation, and is therefore possibly not significant.

\subsection{Giant micropulses?}

Another phenomenon, possibly related to giant pulses, are giant micropulses (Johnston et al. 2001). These are pulses that are not necessarily extreme in the sense that they have a large integrated pulse energy $E$, but their peak flux densities are very large. To investigate this phenomenon the longitude-resolved cumulativeenergy distributions are calculated and the contour levels are shown in the middle panels of Fig. 7 . In these plots the $1 \%$ contour shows the flux density that $1 \%$ of pulses exceed. So at a pulse-longitude bin $i$ the $1 \%$ contour shows that $1 \%$ of all the pulses have (in that single longitude bin $i$ ) a flux density $F_{i}$ that is at least as bright as indicated by the contour. The flux density is compared with the average flux density $\left\langle F_{\mathrm{p}}\right\rangle$ at the pulse longitude of the peak of the pulse profile.

One can see that the highest measured peak flux of a single pulse in the $327-\mathrm{MHz}$ AO-P1 observation is almost $400\left\langle F_{\mathrm{p}}\right\rangle$. In the AO-P2 observation the brightest single pulse (which is also located in the centre of the pulse profile) has a peak flux of $\sim 420\left\langle F_{\mathrm{p}}\right\rangle$. This is an order of magnitude brighter than the giant micropulses observed for the Vela pulsar (Johnston et al. 2001) and PSR B1706-44 (Johnston \& Romani 2002).

The giant micropulses of the Vela pulsar are observed to have nearly complete linear polarization (Johnston et al. 2001) and are limited to the leading edge of the pulse profile. The bright pulse in Fig. 6 (with an integrated energy of $22.9\langle E\rangle$ ) is almost $100 \%$ linearly polarized, resulting in a linear polarization profile that is almost indistinguishable from the total intensity profile. However, in the case of PSR B0656+14 the "giant micropulses" are less confined. Although, like the brightest integrated pulses, the pulses with the largest peak fluxes are limited to the leading and central regions and are not found in the shoulders at the trailing side of the pulse profile.

Yet the bright pulses found in the centre of the profile are not, in relative terms, the most extreme examples of spikes in the emission. For instance at pulse longitude $157^{\circ}$ there is a single pulse that has a peak flux of $32\left\langle F_{\mathrm{p}}\right\rangle$ (see the top middle panel 

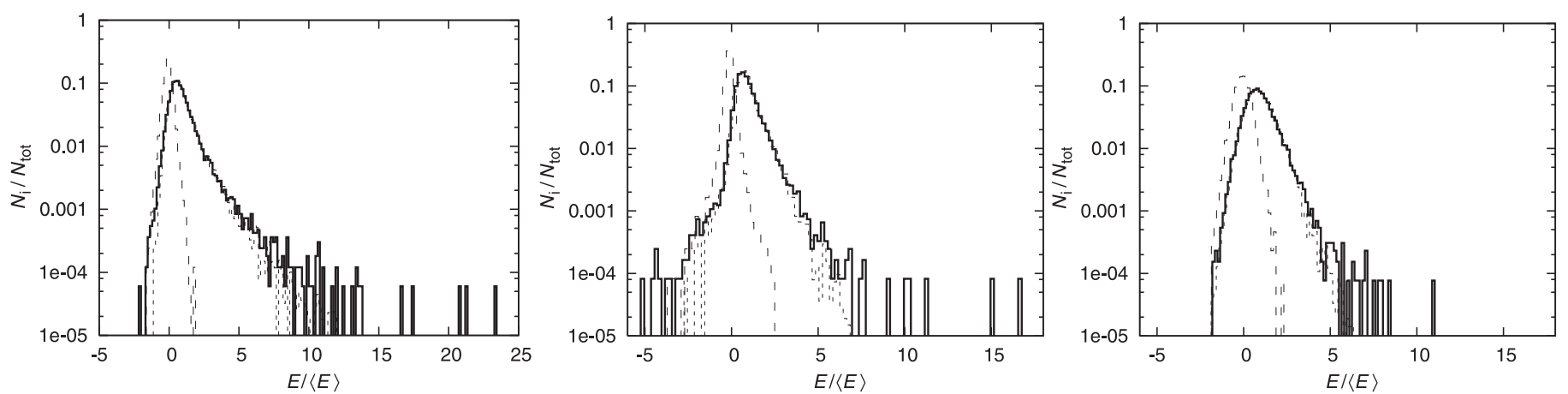

Fig. 5. The pulse-energy distribution (solid line) of (from left to right) the 327-MHz AO-P2, 1525-MHz AO-L and the 1380-MHz WSRT-L observations, together with the fit (dotted line). The off-pulse energy distributions are the dashed lines. The energies are normalized to the averagepulse energy and the two high frequency observations are corrected for scintillation. In the AO-P2 observation there is a pulse of $116\langle E\rangle$, which falls outside the plotted range.
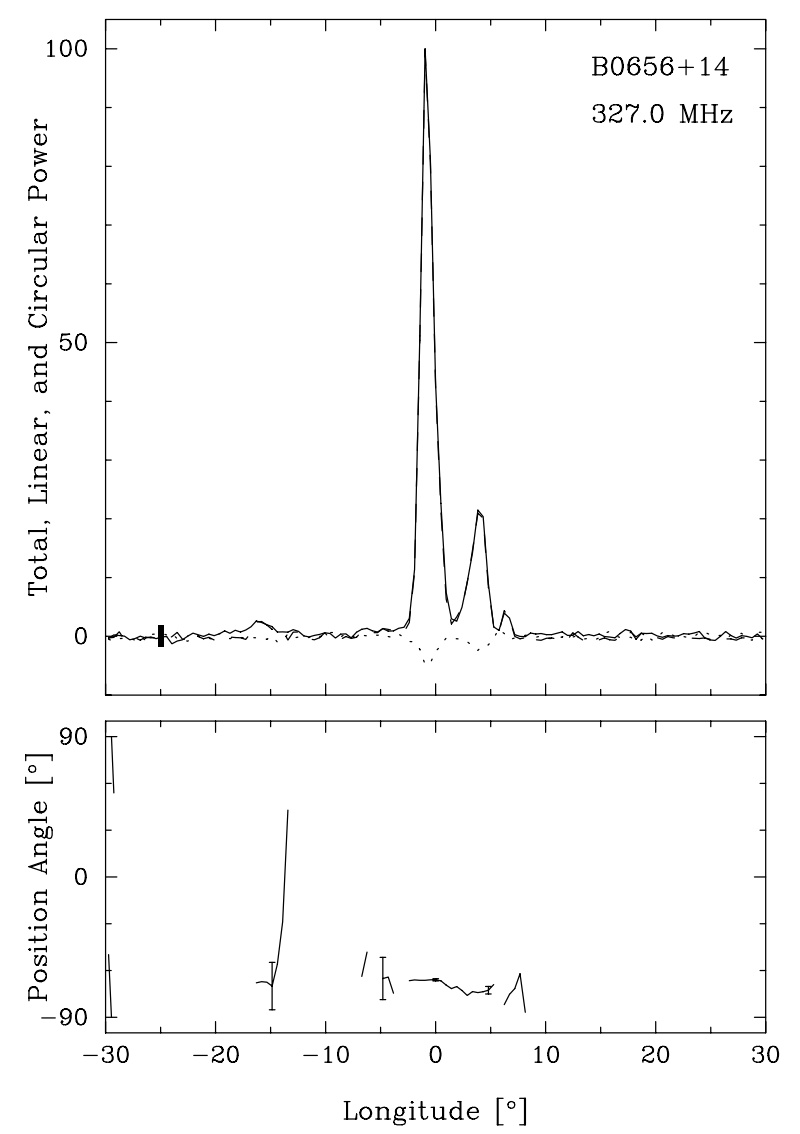

Fig. 6. The top panel shows a typical bright single pulse of the $327-\mathrm{MHz}$ AO-P1 observation in total intensity (solid line), linear polarization (dashed line, but almost indistinguishable from the solid line) and circular polarization (dotted line). The peak-flux of this pulse is normalized to 100 . The bottom panel shows the polarization angle.

of Fig. 7). This is not unusual for the bright pulses in central regions of the profile. However, the peak flux of this pulse is almost 1700 times stronger than the average-pulse intensity $\left\langle F_{\mathrm{i}}\right\rangle$ at that pulse longitude (see right panel of Fig. 7). At the outer edges of the pulse profile the uncertainty in the average-pulse intensity becomes large, making the uncertainties in the relative peak flux on the edges of the right panels of Fig. 7 very large. So for instance the huge peak at pulse longitude $218^{\circ}$ in the top right panel of Fig. 7 is probably caused by a statistical fluctuation that makes $\left\langle F_{\mathrm{i}}\right\rangle$ small, rather than by a bright single pulse. Note that the bright pulse at pulse longitude $157^{\circ}$ is both seen as a spike in the dashed line of the middle panel of Fig. 7 as well as an increase in intensity of the pulse profile.

\subsection{An exceptional pulse}

The most exceptional bright pulse is located on the leading edge of the pulse profile (the bump in the dashed line starting at pulse longitude $143^{\circ}$ in the middle top panel of Fig. 7). This single pulse is plotted in the left panel of Fig. 8. With an integrated pulse energy of $12.5\langle E\rangle$ the pulse is very strong, but not unusual for this pulsar (although it qualifies as a giant pulse). What makes this pulse special is that the pulse is extremely bright compared with the local average-pulse profile intensity. Its peak flux is about 2000 times that of the average-pulse intensity at that location.

Especially because this pulse is found well away from the centre of the profile, it is important to check if this pulse is not generated by RFI. To prove that this pulse is indeed emitted by the pulsar, the pulse is plotted with frequency resolution (middle panel of Fig. 8) and is not de-dispersed. A non-dispersed (Earth related) signal would be visible as a vertical band in this plot, however the dispersion track matches what is expected from the DM of this pulsar (and is the same for the two pulses in the centre of the profile) proving that this exceptional pulse is emitted by the pulsar. Notice also that the effect of scintillation is clearly visible (which is further evidence of the reality of the signal) and that the scintillation bandwidth is much smaller than the bandwidth of the observation (as calculated in Sect. 3.4). Notice also that the scintillation pattern of the bright pulse is the same as that of the two peaks in the middle of the pulse profile.

To emphasize how extreme this pulse is, the longituderesolved energy distribution at the position of the peak of this pulse is calculated (right panel of Fig. 8). The peak flux of the pulse is 31 times higher than the average peak flux of the pulse profile. There is only one other detection of a single pulse at the same pulse longitude (with a flux density of $5.5\left\langle F_{\mathrm{p}}\right\rangle$ ). The remaining 25000 pulses do not have intensities above the noise level. This implies that these two pulses either belong to an extremely long tail of the energy distribution, or that only very sporadic pulses are emitted at this pulse longitude.

\subsection{The pulse-energy distribution}

The pulse-energy distributions of giant pulses can be described by a power law (e.g. Lundgren et al. 1995), while the pulseenergy distribution of "normal" pulses can often be described 

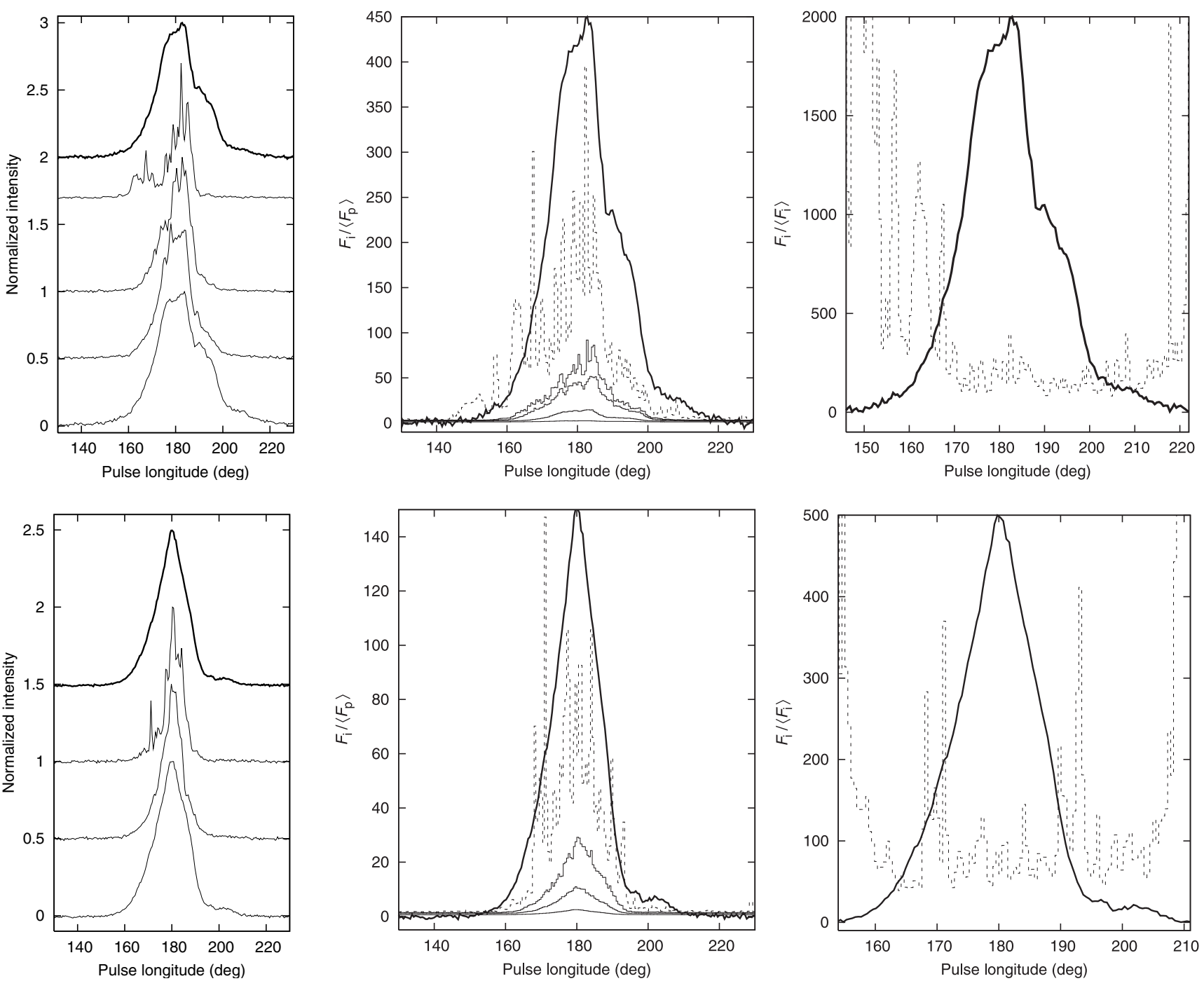

Fig. 7. The top and bottom panels are from the 327-MHz AO-P1 and 1525-MHz AO-L observation respectively. Left: the top thick line is the average-pulse profile. The other profiles are (from top to bottom) the average profile of the pulses with $E>20\langle E\rangle, 10-20\langle E\rangle, 5-10\langle E\rangle$ and $0-5\langle E\rangle$ for the 327-MHz observation and contain 15, 87, 484 and 19926 pulses respectively. For the 1525-MHz observation these profiles are for the pulses with an integrated energy $E>5\langle E\rangle, 2.5-5\langle E\rangle$ and $0-2.5\langle E\rangle$ and contain 39, 462 and 11426 pulses respectively. All the profiles are normalized and given a vertical offset for clarity. Middle: the top thick solid line is the pulse profile. The other lines are contour levels of the longitude-resolved cumulative-energy distribution. The dotted line shows the brightest time sample for each pulse-longitude bin (compared with the average peak flux $\left\langle F_{\mathrm{p}}\right\rangle$ at the pulse longitude of the peak of the pulse profile). The top solid contour level shows the energy of the 10th but brightest sample for each pulse longitude. The others show the energy levels of the cumulative distribution at (from top to bottom) the $0.1 \%, 1 \%$ and $10 \%$ level. Right: the solid line is the pulse profile. The dotted line shows the brightest time sample for each pulse-longitude bin compared with the average-pulse intensity $\left\langle F_{\mathrm{i}}\right\rangle$ at that pulse longitude.

by lognormal statistics (e.g. Cairns 2004; Cairns et al. 2001; Johnston et al. 2001). Because only a fraction of the pulses are giant pulses, the energy distribution of all the pulses consist of two components and a break might appear. From Fig. 5 it is clear that there is no obvious break in the pulse-energy distribution of PSR B0656+14. While no clear breaks are observed in the pulse-energy distributions, a break could be hidden below the noise level. Therefore an important question we should now ask is whether the whole energy distribution (including the part that is below the noise level) can be described by a single distribution, or is a multi-component distribution.

The very bright nature of some of the pulses from PSR B0656+14 may make a contribution to the overall noise received by the telescope and thus the off-pulse noise level would not be an accurate estimator of the on-pulse noise. However, we find that the pulsar signal is sufficiently smeared in time by interstellar dispersion to only increase the overall noise level with a few percent during the brightest recorded pulse. Moreover, the majority of the pulses are much weaker than this particular pulse, so it is appropriate to continue to use the offpulse noise when estimating the $S / N$ of the pulses.

\subsubsection{Fitting procedure of the pulse-energy distribution}

The pulse-energy distribution is modeled with a single lognormal or a power law.

$$
\begin{aligned}
& P_{\text {powerlaw }}(E) \propto E^{p} \\
& P_{\text {lognormal }}(E)=\frac{\langle E\rangle}{\sqrt{2 \pi} \sigma E} \exp \left[-\left(\ln \frac{E}{\langle E\rangle}-\mu\right)^{2} /\left(2 \sigma^{2}\right)\right] .
\end{aligned}
$$



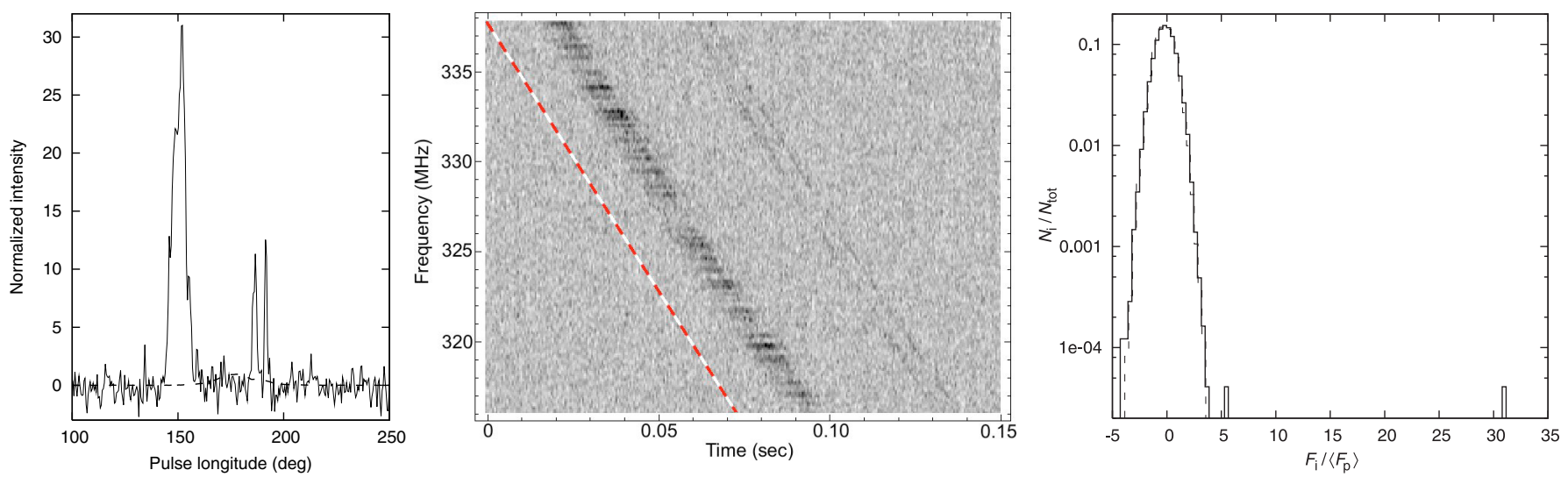

Fig. 8. The exceptional pulse at the leading edge of the pulse profile in the AO-P1 observation. Left: the single pulse (solid line) compared with the average of all the pulses (dashed line). The fluxes are normalized such that the peak of the average pulse is 1 . Middle: the same pulse, but now with frequency resolution (non-dedispersed). The dashed line shows the expected dispersion track for the DM of this pulsar. Right: the longitude-resolved energy distribution at the longitude of the peak of the strong pulse (solid line) and the off-pulse distribution (dashed line).

Table 2. The parameters of the best fits to the pulse-energy distributions of the four observations. Besides the parameters of the fitted distribution (where $f_{\text {weak }}=N_{\text {weak }} / N_{\text {tot }}$ is the fraction of weak pulses that are added to the distribution), also the total $\chi^{2}$, the number of degrees of freedom and the significance probability are tabulated.

\begin{tabular}{c|cccccc}
\hline \hline REF & $\sigma$ & $\mu$ & $f_{\text {weak }}$ & $\chi^{2}$ & $N_{\text {d.o.f }}$ & $P\left(\chi^{2}\right)$ \\
\hline [2mm] AO-P1 & 0.99 & -0.34 & $13 \%$ & 105 & 32 & $1 \times 10^{-9}$ \\
AO-P2 & 0.79 & -0.34 & $0 \%$ & 146 & 24 & $2 \times 10^{-19}$ \\
AO-L & 0.56 & -0.13 & $0 \%$ & 240 & 44 & $8 \times 10^{-29}$ \\
WSRT-L & 0.48 & -0.03 & $8 \%$ & 148 & 44 & $4 \times 10^{-13}$ \\
\hline
\end{tabular}

Because the integral of a power-law distribution is infinite, a cutoff energy $E_{\min }$ (the minimum energy of the pulses) must be introduced. So the power-law distribution, as well as the lognormal distribution, are defined by two fit parameters: $p$ and $E_{\min }$ in the case of a power-law distribution and $\mu$ and $\sigma$ in the case of a lognormal distribution.

The possible existence of two distributions, one of strong and one of weak pulses, is modeled by adding pulses with zero energy to the model distribution. It is important to note that these pulses with zero energy are not necessarily representing real "nulls". They represent a distribution of pulses that are significantly weaker than the tail of strong pulses. This distribution of weak pulses can be described by only a single parameter: the fraction of weak pulses $\left(f_{\text {weak }}=N_{\text {weak }} / N_{\text {tot }}\right)$.

The three model parameters are not independent of each other. They are coupled by the requirement that the averagepulse energy $\langle E\rangle$ of the model distribution should match the observed value. This constraint is used to fix the fraction of weak pulses for a given combination of the other two fit parameters. Although this description of the pulse-energy distribution is probably oversimplified, it has the advantage that there are only two fit parameters. Given the $S / N$ ratio of the observations, the addition of more fit parameters would probably over-interpret the available information.

To take into account the effect of noise, the model energy distribution is convolved with the noise distribution. Because of the presence of some RFI, the noise distributions of the AO observations deviate from a pure Gaussian distribution. Therefore the observed noise distribution is used rather than a theoretical noise distribution. For a given set of fit parameters, the model pulseenergy distribution is compared with the observed distribution. The fit parameters are optimised by minimizing the $\chi^{2}$ using the downhill simplex method (amoeba algorithm; Press et al. 1986). Fitting is restricted to bins with more than 10 counts and counting statistics are used for the measurement uncertainties in each bin. The fitting depends on the binning used, and therefore we checked that all results are valid for different binning and count limits.

\subsubsection{Results of fitting the pulse-energy distributions}

For all four observations we fit a cutoff power law and a lognormal distribution (convolved with the observed noise distribution) to the pulse-energy distribution. As described above we include the possibility for a separate distribution of weak pulses. In all cases the fits using a cutoff power law are worse than the fits with a lognormal distribution. The results of the fits with a lognormal distribution are summarized in Table 2 and in Fig. 5 the fits (convolved with the noise distributions) are shown overlaid over the observed distributions. As one can see the fits can qualitatively describe the observations well.

The significance of the best fits are low, indicating that the model is oversimplified. Furthermore, the best fits for the different observations at the same frequency are quite different. For instance the fit to the AO-P1 data is significantly improved by adding a distribution of weak pulses while the addition of weak pulses to the AO-P2 data does not improve the fit. This probably indicates that the observations are too short to get a pulse-energy distribution that is stable. This may be related to the gradual profile change described in Sect. 3.3 as the fits for the first and second halves of the AO-P2 observation are different. The fit for the first half of the observation (when the central peak is relatively bright) is improved by adding a distribution of weak pulses, while this is not the case for the second half of the observation.

The pulse-energy distribution of PSR B0656+14 can (at least for some time intervals) only be described by a multicomponent distribution consisting of a distribution of strong pulses and weak pulses. The absence of a clear break in the observed distribution may indicate that the transition from weak to strong pulses is smooth. If a sharp break exists, it must be hidden below the noise. 

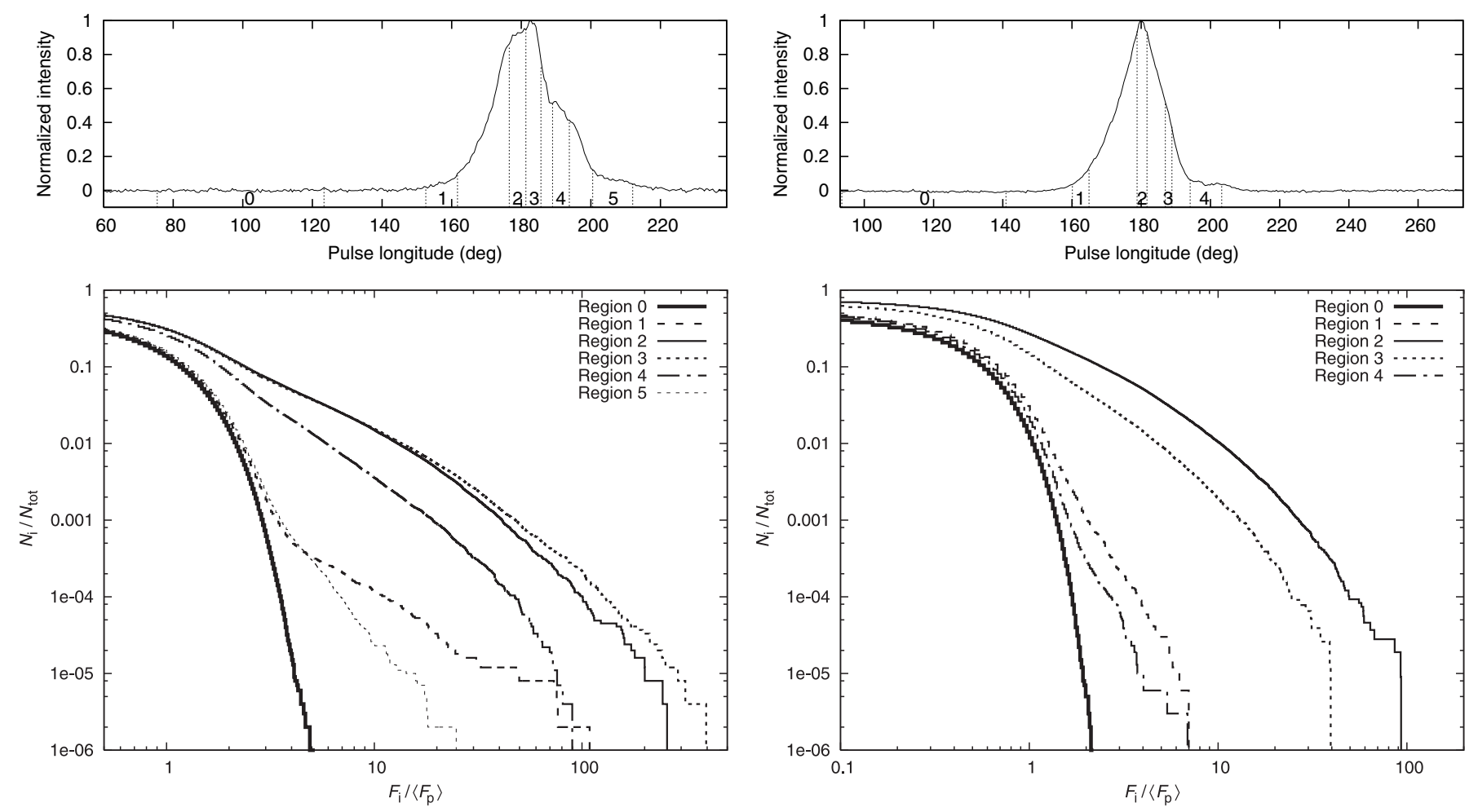

Fig. 9. The longitude-resolved cumulative-energy distribution for different regions (between the lines) in the pulse profile. The left and right panels are for the $327-\mathrm{MHz}$ AO-P1 and the $1525-\mathrm{MHz}$ AO-L observations respectively.

\subsubsection{The longitude-resolved energy distribution}

In Fig. 9 examples of longitude-resolved cumulative-energy histograms are shown. These are the energy histograms of single pulse-longitude bins. For better statistics, energy histograms of multiple successive pulse-longitude bins in a small longitude range are added. The regions used are shown in Fig. 9 as well. It is interesting to see the difference in the cumulative distribution of region 1 and 5 of the $327-\mathrm{MHz}$ AO-P1 observation. Although the regions are comparable in intensity in the averagepulse profile, the leading side of the profile shows much brighter spikes. As for for the pulse-energy distributions, the longituderesolved energy distributions do not show a clear break. The apparent break in the longitude-resolved energy distributions at the cutoff energy of the noise distribution (best visible for region 1 in the bottom left panel of Fig. 9) is most likely not a physical break in the energy distribution, but just the transition from noise-dominated to a pulse-dominated energy range.

Using exactly the same method as used to fit the pulseenergy distribution we tried to fit the longitude-resolved energy distributions. Again it turns out that a cutoff power law cannot describe the data well, but a lognormal distribution can be fit with much more confidence. For instance, the longitude-resolved energy distribution of the peak of the AO-P2 observation can be described well with a lognormal distribution $(\mu=-1.5$ and $\sigma_{\text {lognorm }}=1.7$, without a distribution of weak pulses). As one can see in Fig. 10, the fit describes the data qualitatively very well. The total $\chi^{2}=104$ with 61 degrees of freedom $\left(P\left(\chi^{2}\right)=5 \times 10^{-4}\right)$. This is a better fit than those found for the integrated pulse-energy distribution, which is surprising because the longitude-resolved energy distribution has better statistics because of two reasons. Firstly, the distributions of 7 bins were added which makes the counting uncertainties smaller. Secondly, the longitude-resolved distribution is much more

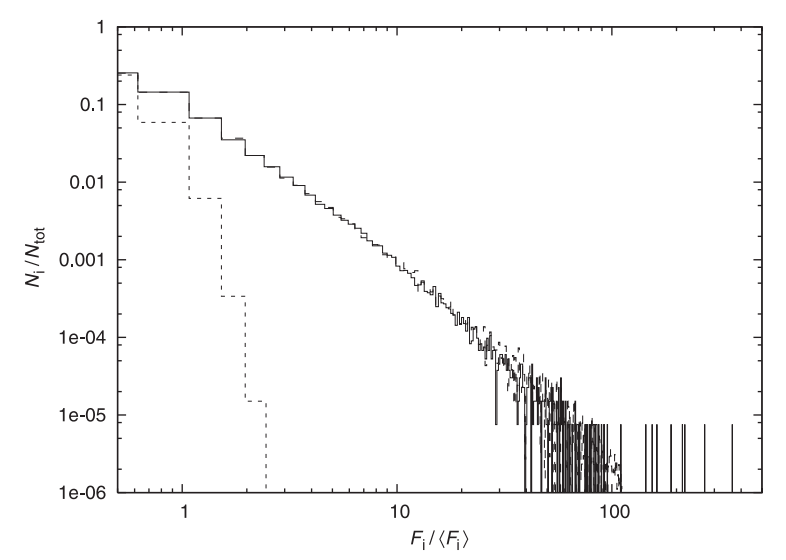

Fig. 10. The tail of the longitude-resolved energy distribution of the peak of the 327-MHz AO-P2 observation (solid line), the fit (dashed line) and the off-pulse distribution (dotted line).

extended (compared with the off-pulse distribution) than the integrated pulse-energy distribution.

\subsubsection{Two populations of pulses?}

There is no direct evidence for the existence of a separate energy distribution for the strongest pulses of PSR B0656+14 as there is no clear break in the pulse-energy distribution. Nevertheless there is evidence from the energy distributions that the bursts and the underlying weak emission have different properties.

The first argument is based on the frequency evolution of the pulse-energy distribution. Although the exact shape of the pulse-energy distribution remains unclear, it is steeper and less extended at high frequencies ( $\sigma$ is smaller). The difference in shape of the distributions at the two frequencies is not only a 

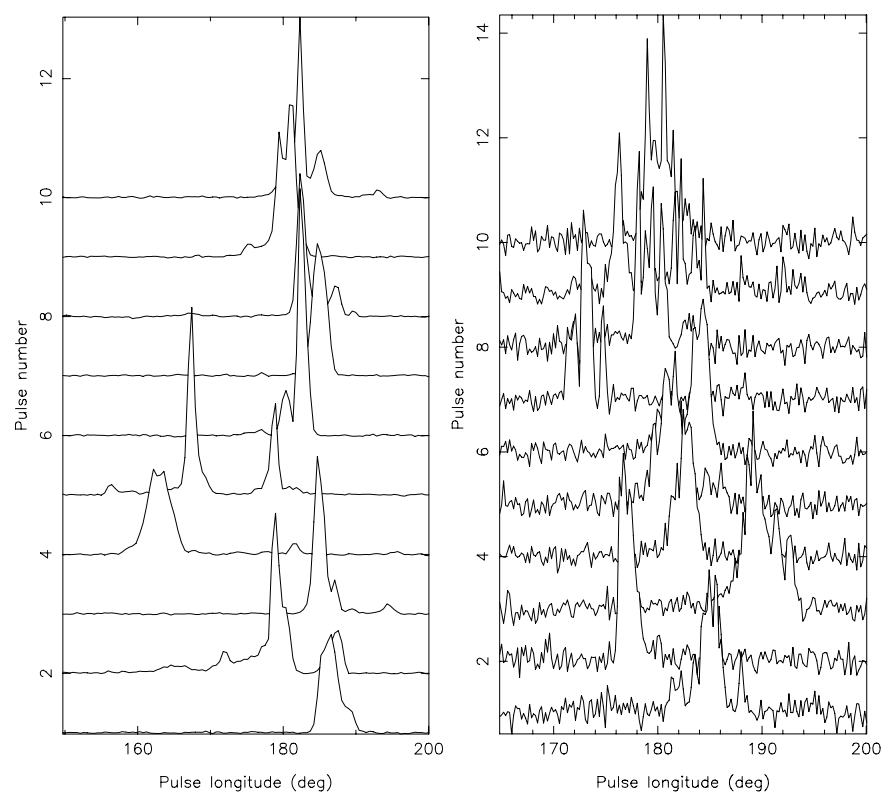

Fig. 11. The ten brightest pulses of the 327-MHz AO-P1 observation (left panel) and of the 1380-MHz WSRT-L observation (right panel).

scaling in energy range. The energy distribution of the high frequency observation is too steep to be scaled to fit the $327-\mathrm{MHz}$ observations, which could be because the emission shows more bright pulses at low frequencies.

Secondly, as discussed above, the longitude-resolved distribution is fitted much better by a lognormal distribution than the integrated pulse-energy distribution. This is probably because when a single pulse contains a very bright subpulse, it is more likely to contain more bright subpulses (see for example Fig. 11). This affects only the integrated energy. This is therefore evidence that the bursts of radio emission not only cluster in successive pulses, but they also tend to cluster within the pulses. This clustering generates a tail in the pulse-energy distribution which is relatively more extended than that of the longitude-resolved energy distribution. This also explains why all observations show a flattening in their integrated pulse-energy distribution (containing only a few pulses) at the highest energies that cannot be fit (see Fig. 5).

Thus we have to accept the perhaps surprising conclusion that, unlike the energy distributions of pulsars with true "giant" pulses, those of PSR B0656+14 offer no direct evidence of comprising two components. Although it is possible that the bright and weak pulses are associated with the extreme ends of a single smooth energy distribution, we will demonstrate in the next section that they have very different characteristics.

\section{Characteristics of the spiky and weak emission}

\subsection{Appearance of the spiky emission}

Perhaps the strongest argument for the presence of a distinctive "spiky" emission is the appearance of the pulse stack itself (Fig. 4). Bright, narrow, subpulses seem to dominate the emission. This suggests that they may have different properties from the rest of the emission. In the left panel of Fig. 11 the ten brightest pulses of the AO-P1 observation are shown. One can see that the spikes are not only narrow, but appear to be quasi-periodic. In the left panel of Fig. 11 one can see that most strong pulses show a $\sim 11 \mathrm{~ms}\left(10^{\circ}\right)$ periodicity (for instance pulse 5 ). In the right panel of Fig. 11, the ten brightest pulses of the WSRT-L
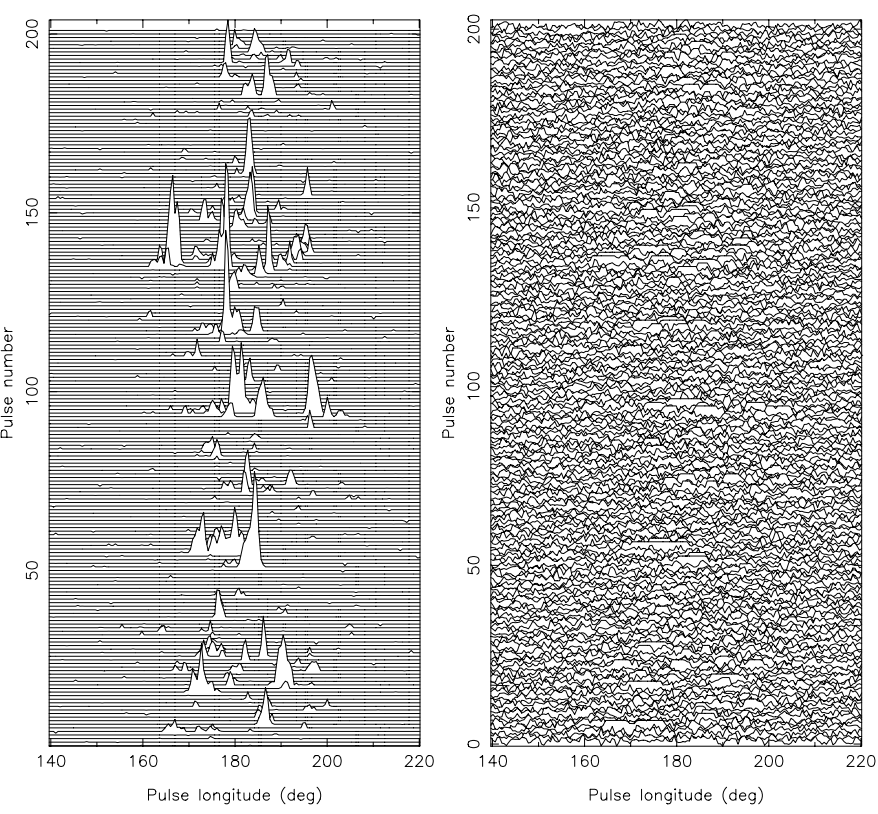

Fig. 12. Here the same 200 successive pulses of the 327-MHz AO-P1 observation as plotted in Fig. 4 are shown, but separated into the spiky (left) and weak (right) emission.

observation are shown, which has a higher time resolution allowing the detection of structure on shorter timescales. In addition to the $11-\mathrm{ms}$ periodicity, a $1-\mathrm{ms}\left(1^{\circ}\right)$ periodicity is also revealed (for instance pulse 9). In the rest of this section we will further characterize the spiky and weak emission in detail.

\subsection{Separation of the spiky and weak emission}

Although the pulse sequence of Fig. 4 is dominated by the very apparent spiky emission, this is accompanied by an almost indiscernible background of weak emission. To separate these two components of the emission, we have applied an intensity threshold to the data. The intensities of the time samples in the pulse stack of the weak emission are truncated if they exceed this threshold. The time samples in the pulse stack of the spiky emission contains only samples with intensity in excess of this threshold. When the pulse stack of the weak emission is added to the pulse stack of the spiky emission, one retrieves exactly the original pulse stack.

Because no clear break is observed in the longitude-resolved energy distribution (Fig. 9), we have set the threshold intensity such that $99 \%$ of the noise samples are below this threshold value. Only about $1 \%$ of the weak emission is expected to be classified as spiky emission due to noise fluctuations, so the emission classified as spiky is expected to be almost pure. Not only do the noise fluctuations make it impossible to completely separate the weak and spiky emission, it is also very well possible that the energy distributions of the two components overlap. In Fig. 12 one can see the pulse stack obtained by the separation of the spiky and weak emission for exactly the same pulse sequence as shown in Fig. 4. The integrated power of this sequence of weak pulses is about 3 times greater than that of the sequence of the spiky emission. This shows that a significant fraction of the pulsar's emission lies at or below the noise level.

By linking the threshold intensity to the noise level, our analysis depends on the sensitivity of the telescope. An alternative natural choice is to stipulate that the integrated weak and spiky emission profiles have equal power. This resulted in an intensity 

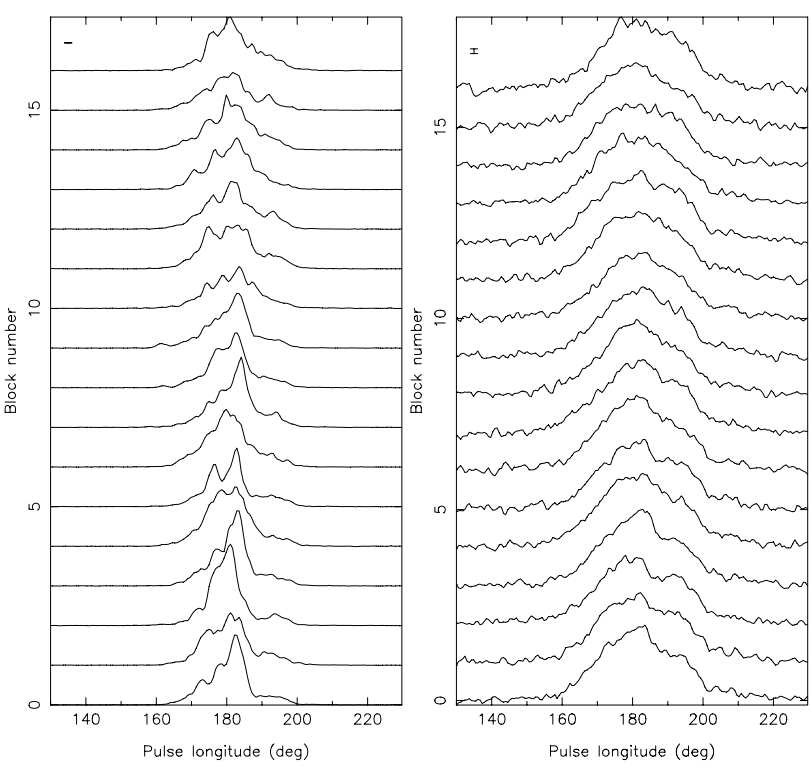

Fig. 13. The pulse profiles obtained by averaging successive blocks of one thousand pulses each of the 327-MHz AO-P2 observation (like the middle right panels in Fig. 2), but now for the spiky and weak emission separately. Note the gradual changes in the spiky emission profile, in contrast to the steady weak emission. The 1-sigma error bars are plotted in the top left corner.

threshold very close to the one described above. In general, we found that our conclusions did not depend on the exact choice of threshold intensity. This also implies that the effects of interstellar scintillation in the L-band observations, for which we have made no correction, will not significantly alter our results.

\subsection{Stability of the pulse profile revisited}

The reason why the pulse profile is unstable at low frequencies (see Fig. 2) is the presence of the spiky emission. These spikes have a very uneven longitude distribution and therefore many pulses are required to obtain a steady profile. This is demonstrated in Fig. 13, where the profiles of successive blocks of one thousand pulses each are plotted separately for the spiky and weak emission. One can clearly see that it is the spiky emission that is highly unstable, whereas the weak emission converges rapidly to a stable profile. Moreover in this plot one can see that the observed gradual change in the pulse profile in the AO-P2 (middle right panel of Fig. 2) is caused by a gradual change in the spiky emission while the weak emission remains the same. Around pulse 11000 (block 11) the profile of the spiky emission evolves from a peaked profile to a broader profile that resembles more the shape of the profiles of the weak emission. The fact that the profile of the spiky emission can evolve independently of the weak emission is an additional argument that PSR B0656+14 has two kinds of emission. This also makes, as discussed in Sect. 3.3, an instrumental effect a very unlikely source for the observed profile shape changes.

\subsection{Profiles of the spiky and weak emission}

The pulse profiles of the separated spiky and weak emission are shown in Fig. 14. At both frequencies (left and middle panels) there is a striking difference in shape between the spiky and weak emission: the spiky emission profile is significantly narrower more pointed than the total pulse profile, in contrast to the flatter bell-like shape of the weak profiles. This is consistent with the longitude-resolved energy distribution (Fig. 7) that shows that the strongest spikes are in the central part of the pulse profile. At $327 \mathrm{MHz}$ (left panel) the profile is generated by a mix of both spiky and weak emission, but the ratio of their intensities is different in the different profile components. At $1525 \mathrm{MHz}$ (middle panel) the profile of the spiky emission is an almost perfect isosceles triangle, while the weak emission is much less symmetric and only roughly triangular. At both frequencies the shoulder starting at pulse longitude $200^{\circ}$ is completely generated by weak emission.

In the right panel of Fig. 14 we have overlaid the total, spiky and weak emission profiles of the AO-P1 and AO-L observations. As in Fig. 1, the centroid of the central component is fixed at $180^{\circ}$ and the profiles are scaled in intensity such that the central component overlaps. Neither the spiky nor the weak emission appears to evolve at the leading edge, and the broadening of the profiles at low frequencies of both the spiky and weak emission is only due to the appearance of more emission components.

\subsection{The modulation-index profiles}

To characterize the modulation properties we have used the Discrete Fourier Transform (DFT) to calculate the Longitude Resolved Fluctuation spectrum (LRFS; Backer 1970), which is used to calculate the longitude-resolved modulation index (Edwards \& Stappers 2002, 2003). The LRFS is obtained by taking DFTs along lines of constant pulse longitude in the pulse stack. The resulting spectra (bottom panels of Fig. 15) have pulse longitude on the horizontal axis and $P_{1} / P_{3}$ on the vertical axis. Here $P_{3}$ is the subpulse pattern repetition rate and $P_{1}$ the pulse period.

The longitude-resolved variance $\sigma_{\mathrm{i}}^{2}$ is computed by vertical integration of the LRFS from which the longitude-resolved modulation index $m_{\mathrm{i}}=\sigma_{\mathrm{i}} / \mu_{\mathrm{i}}$ is derived (where $\mu_{\mathrm{i}}$ is the average intensity at longitude bin $i$. For more details we refer to Weltevrede et al. (2006a). As one might expect from the spiky nature of the emission of this pulsar, its modulation index is unusually high, especially at low frequencies. The modulation index increases from slightly below 2 at the leading edge of the profile to about 3 at the trailing edge in the $1525-\mathrm{MHz}$ AO-L observation (right panel of Fig. 15). The modulation-index profile of the 1380-MHz WSRT-L observation (not shown) is very similar, but slightly lower probably due to a small amount of residual RFI in the AO observations. Notice also that the spectra of both AO observations, despite the large number of pulses, are still "contaminated" by a few very bright pulses causing vertical stripes in the LRFSs and spikes in the modulation-index profiles. At $327 \mathrm{MHz}$ the modulation-index profile has evolved to a "W-shape" which varies between 2 and 5 (left panel of Fig. 15).

In Fig. 16 the modulation-index profiles are shown for the separated spiky and weak emission. The separation shows that the modulation index of the spiky emission is higher and that of the weak emission lower compared with the modulation index of the total emission. The precise degree of this effect depends strongly on the threshold intensity used for the separation. Therefore the shape of the modulation-index profiles is more interesting than its exact magnitude. To make the comparison in shape more clear in Fig. 16, we scaled-down the modulation-index profile of the spiky emission. It is striking that the modulation profiles of both the spiky and weak emission at both frequencies have a much simpler U-shape compared with the modulation index of all the emission. A U-shape is usual for profile components (Weltevrede et al. 2006a), suggesting that 

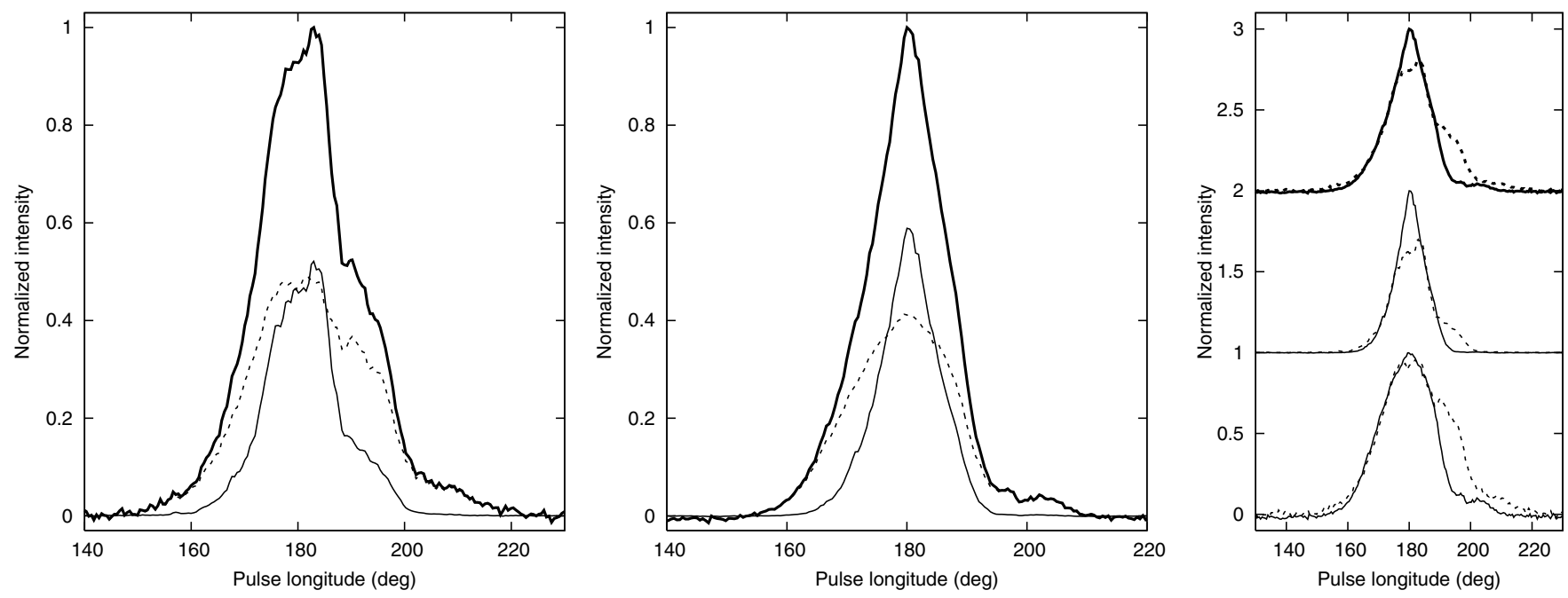

Fig. 14. Left: the normalized total emission pulse profile (thick solid line), which is decomposed into the spiky (thin solid line) and weak emission profile (dotted line) for the 327-MHz AO-P1 observation. Middle: same as the left panel, but for the 1525-MHz AO-L observation. Right: the profiles of the total, spiky and weak emission with a vertical offset of 2, 1 and 0 respectively of the AO-P1 (solid lines) and AO-L observation (dotted lines). The peaks of the profiles of the AO-L observation are normalized and those of the AO-P1 observation are scaled such that the central peaks fit each other.
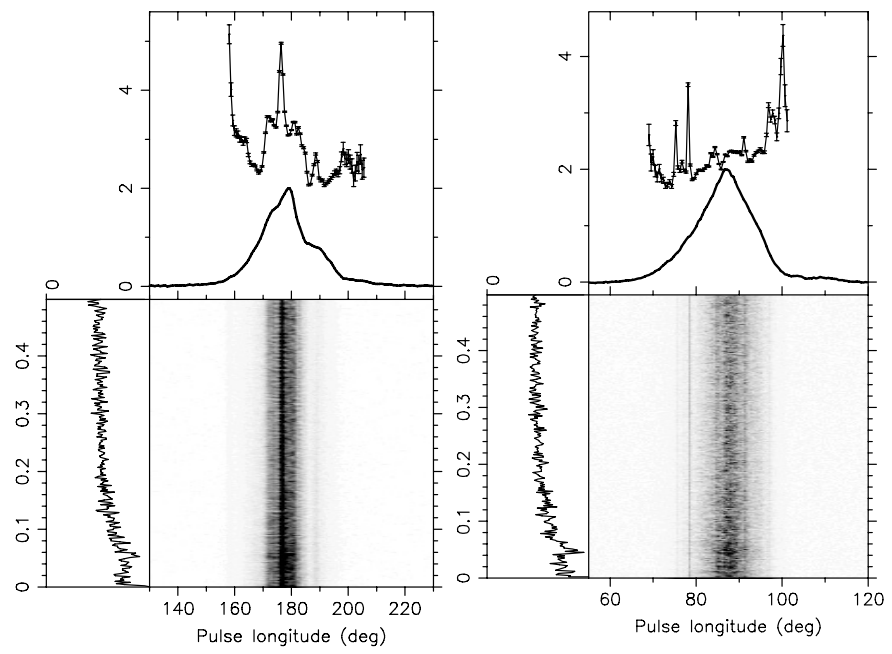

Fig. 15. The modulation properties of PSR B0656+14 in the 327-MHz AO-P2 (left) and 1525-MHz AO-L (right) observation. The top panel shows the integrated profile (solid line) and the longitude-resolved modulation index (solid line with error bars). Below this panel the LRFS is shown. The power in the LRFS is horizontally integrated, producing the side-panel.

the profiles of the weak and spiky emission can be regarded as true (overlapping) profile components. With this in mind we can understand the complex shape of the modulation profiles of the total emission in Fig. 15.

Because the profile of the weak emission is broad, the edges of the profile are dominated by the weak emission at $327 \mathrm{MHz}$ (see left panel of Fig. 14). This causes the outer edges of the modulation-index profile to flare out at this frequency, like the modulation-index profile of the weak emission (see Fig. 16). In the central part of the profile a significant fraction of the total emission is spiky emission, so that the local increase in the modulation index makes the modulation-index profile of the total emission $\mathrm{W}$-shaped.

At $1525 \mathrm{MHz}$ the situation is slightly different because the centroid of the profile of the weak emission has an offset toward
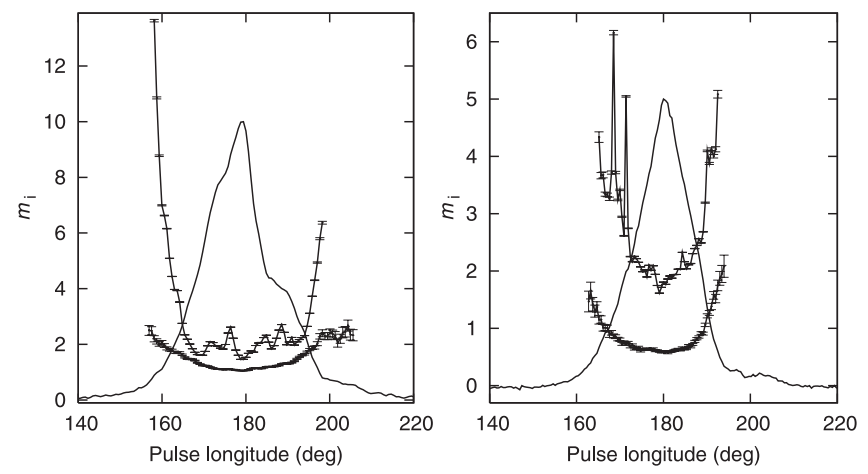

Fig. 16. The left and right panels show the 327-MHz AO-P2 observation and $1525-\mathrm{MHz} \mathrm{AO}-\mathrm{L}$ observation respectively. The modulation-index profiles of the spiky emission (top) and weak emission (bottom) are superimposed on the pulse profiles. The shapes of the modulation-index profiles are important, while their magnitudes have little physical meaning. Therefore the modulation-index profiles of the spiky emission are scaled-down to make the profiles of comparable magnitude.

the leading side of the pulse profile, while the centroid of the profile of the spiky emission is exactly in the centre of the profile (see middle panel of Fig. 14). Hence the weak emission dominates the leading edge, but less so on the trailing edge. This fact, in combination with a relative low modulation index of the spiky emission, causes the otherwise expected $\mathrm{W}$-shape of the modulation-index profile of the total emission to be blurred out by the flaring of the modulation-index profile of the weak emission on the trailing side of the profile.

\subsection{The quasi-periodic modulation}

In the LRFSs (bottom panels of Fig. 15) one can clearly see a $P_{3}=20 \pm 1 P_{1}$ modulation feature, similar to that observed by Weltevrede et al. (2006a). The relatively large uncertainty in $P_{3}$ reflects the fact that this quasi-periodic modulation is weak compared with the non-periodic intensity modulation (in the side panels of the spectra in Fig. 15 there is only a relatively weak and broad feature around $\left.P_{1} / P_{3}=0.05\right)$. Such a feature could be 

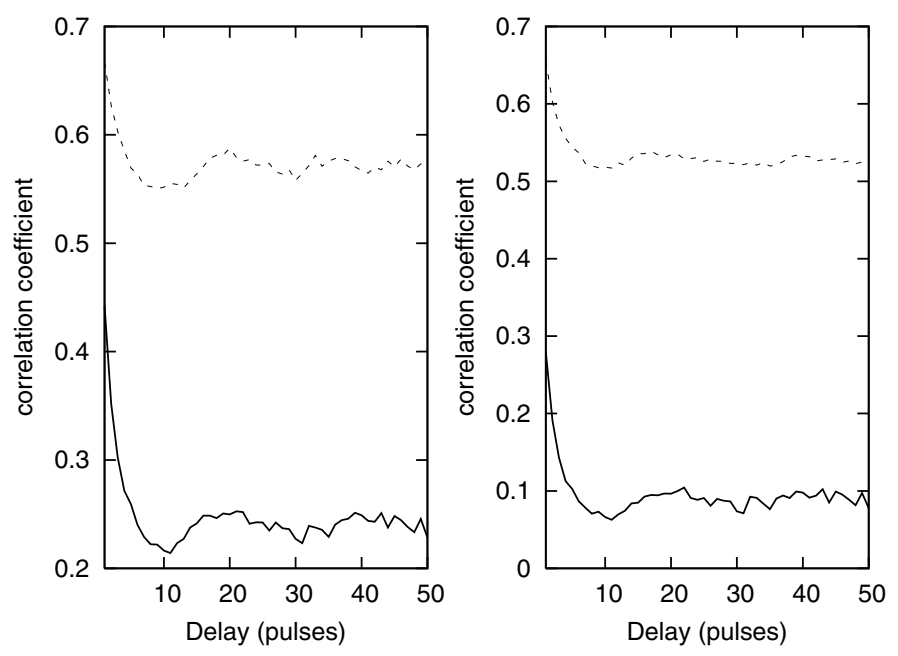

Fig. 17. Left: the ACF of the sequence of pulse energies in the $327-\mathrm{MHz}$ AO-P1 observation (solid line) and the 1380-MHz WSRT-L observation (dotted line). Right: the ACF of the sequence of pulse energies for the spiky emission (solid line) and the weak emission (dotted line) in the 327-MHz AO-P1 observation.

caused by an intensity modulation or a phase modulation (drifting subpulses). To distinguish between these possibilities, we calculated the Two-Dimensional Fluctuation Spectrum (2DFS, Edwards \& Stappers 2002). The 2DFS is obtained by taking DFTs along lines with different slopes in the pulse stack. For a more comprehensive description of the methods used we refer to Weltevrede et al. (2006a). There is no evidence, in any of the four observations, that there exists a preferred drift sense. A preferred negative drift sense has been reported by Backus (1981) at $430 \mathrm{MHz}$, but we suspect that was an artifact of a too short sequence of pulses. We therefore conclude that the modulation features are the result of an intensity modulation on a timescale of $P_{3}=20 \pm 1 P_{1}$ with the phase shifts between the individual pulses essentially random.

To further explore this modulation feature, we calculated the auto-correlation function (ACF) of the sequence of pulse energies (left panel of Fig. 17). Both the 327-MHz AO-P1 and the $1380-\mathrm{MHz}$ AO-L observation show a dip in the ACF at a delay of $\sim 10 P_{1}$. Note that the ACFs of all observations show some evidence for a slight increase at a delay of $\sim 20 P_{1}$ and a decrease at a delay of $\sim 30 P_{1}$. This argues that there is a weak quasiperiodicity with a period of $\sim 20 P_{1}$ (which can just be discerned in the pulse stack Fig. 4). Interestingly, positive correlation with a delay of $\sim 20 P_{1}$ is weak in comparison with the dip at a delay of $\sim 10 P_{1}$. This implies that a burst of strong emission is not necessarily followed $\sim 20 P_{1}$ later by another strong burst. Rather it shows that the burst has decayed within $\sim 10 P_{1}$. Therefore the $P_{3} \sim 20 P_{1}$ feature that appears in the LRFS is only partially associated with a periodic signal, but mostly with a characteristic timescale of the rise and decay of the bursts (i.e. the bright pulses tend to cluster as we discussed in Sect. 4). The ACF of the WSRT-L observation (as well as that of the not shown AO-L observation) is flatter, which shows that at high frequencies there are less very bright pulses and that the clustering of the bright pulses in a few successive pulses is less.

In the right panel of Fig. 17 one can see that the dip in the ACF at a delay of $\sim 10 P_{1}$ is visible both in the spiky and the weak emission. We attempted to test the hypothesis that only the spiky emission, and not the weak emission, is responsible for the dip. However, as the technique used could not wholly
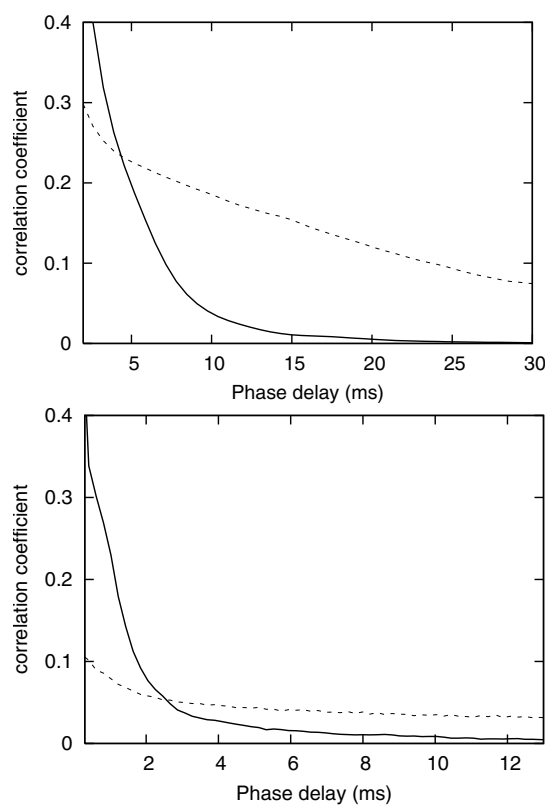

Fig. 18. The average ACF of the single pulses of the spiky emission (solid line) and the weak emission (dotted line) in the 327-MHz AO-P2 (top) and 1380-MHz WSRT-L observation (bottom).

separate the spikes from the weak emission, we could not tell whether the dip in the ACF of the weak emission is caused by the spiky emission mixed in the weak emission, or is an intrinsic property of the weak emission. Nevertheless the dip in the ACF of the spiky emission proves unambiguously that clusters of such emission are sustained over a timescale of $\sim 10 P_{1}$.

\subsection{The time structure of the individual pulses}

In Fig. 11 it is evident that the brightest pulses have timestructures on two timescales. When multiple spikes appear in a single pulse, they appear to be quasi-periodic with a timescale of the order of $10 \mathrm{~ms}$ (this is clearest in the left panel of Fig. 11). The spikes themselves show micro-structure on a timescale of about $1 \mathrm{~ms}$ (only visible in the right panel of Fig. 11). However, we need to know if these timescales are typical for all emission and whether this points to further differences between spiky and weak emission. We therefore calculated the ACF of the single pulses and averaged them over each of our four long observations.

In Fig. 18 the resulting ACFs for the AO-P2 (top) and WSRT-L observation (bottom) are shown. The 1-ms microstructure is clearly visible as a bump in the ACF of the spiky emission in the WSRT-L observation. These are unresolved in the AO observations because of the longer sampling time. At a delay of about $2 \mathrm{~ms}$ the ACF of the WSRT-L observation flattens out, showing that the spikes become unresolved on a timescale of a few ms (i.e. the typical width of the spikes is a few ms). In the AO-P2 observation one can see no clear bump in the ACF of the spiky emission at a delay around $10 \mathrm{~ms}$, so this value cannot be typical. In shorter stretches of data the ACF shows various timescales for the separation between the spikes, which are smeared out in the ACF of the whole observation in the range of about $5-10 \mathrm{~ms}$. Therefore there seems to be no fixed timescale for this periodicity that is stable over a timescale of hours.

Both observations in Fig. 18 show that at short delays the weak emission correlates more weakly than the spiky emission, but on longer timescales ( $\gtrsim 3-5 \mathrm{~ms}$ ) this is reversed. The fact that 
the ACFs of the weak emission are very flat on all timescales demonstrates again that the weak emission of a single pulse is distributed over large parts of the profile width, yielding a large cover fraction. This is further support for the conclusion (as suggested in Sects. 5.2 and 5.3) that the PSR B0656+14's emission can be characterized as a combination of weak emission with a broad longitude coverage across the profile and thinlydistributed bright spikes.

\section{Discussion and conclusions}

\subsection{Comparison with the known population of pulsars}

We have shown that PSR B0656+14 intermittently emits pulses that are extremely bright compared to normal pulsars and with pulse energies well above $10\langle E\rangle$ these pulses formally qualify as giant pulses. Nevertheless these pulses differ from giant pulses and giant micropulses in important ways:

- The pulse-energy distribution of PSR B0656+14 does not show a power-law tail (this is possibly also true for PSR B1133+16; Kramer et al. 2003).

- The widths of the bright pulses of PSR B0656+14 are much greater than those of the extremely narrow giant pulses (e.g. Soglasnov et al. 2004), although similar to that of the giant micropulses.

- The brightest pulses of PSR B0656+14 are an order of magnitude brighter (both in integrated energy and in peak flux) than the giant micropulses of the Vela pulsar (Johnston et al. 2001) and PSR B1706-44 (Johnston \& Romani 2002).

- The bright pulses of PSR B0656+14 are not strongly constrained in pulse longitude. In most other pulsars, both fast (e.g. B1937+21, B1821-24) and slow (e.g. B0031-07) the pulse longitudes where giant pulses appear are highly constrained, whether towards one edge or centrally (e.g. Cognard et al. 1996; Kinkhabwala \& Thorsett 2000; Romani \& Johnston 2001; Kuzmin \& Ershov 2004). Also the giant micropulses detected for the Vela pulsar and the young pulsar B1706-44 are highly confined (in both cases to the leading edge of the pulse profile).

- The claimed empirical correlation between the appearance of giant pulses and high light-cylinder magnetic field strengths clearly fails for PSR B0656+14 since its value (770 Gauss) is well below those of most pulsars exhibiting giant pulses (around $10^{5}$ Gauss, Cognard et al. 1996). However, PSR B0031-07 and a number of other slow pulsars also easily fail this test, so the correlation may only be valid for millisecond pulsars.

- The bright pulses of B0656+14 do not coincide with any of the observed X-ray peaks, in contrast to those of the wellknown pulsars exhibiting giant pulses.

Despite the differences between the spiky emission of PSR B0656+14 and the giant micropulses listed above, there are also interesting parallels between PSR B0656+14 and Vela. The pulse profile of PSR B0656+14 has a shoulder on the trailing edge reminiscent of a similar feature discovered in Vela (Johnston et al. 2001) and B1937+21 (Cognard et al. 1996). In both, the shoulder is not associated with the giant pulses (Kinkhabwala \& Thorsett 2000), and that also appears to be the case in PSR B0656+14. Furthermore both pulsars have lowfrequency intensity modulations and neither pulsar shows a coincidence between the locations of the radio and high-energy emission.
Many of the exceptional properties of PSR B0656+14 have led us to point out (Weltevrede et al. 2006b) that this pulsar, were it not so near, could have been discovered as an RRAT. Chief among these are the infrequent, but luminous pulses with very high peak fluxes and widths comparable with those of the RRATs. Furthermore, like RRATs, PSR B0656+14 has a lightcylinder magnetic field strength which is not exceptional.

Surprisingly, the middle-aged PSR B0656+14 also shares many properties with recycled millisecond pulsars. For instance both have wide profiles that have a more or less constant width or component separation over a very wide frequency range (e.g. Kramer et al. 1999a) ${ }^{2}$. Furthermore the emission of millisecond pulsars is usually highly polarized with a flat linear polarization profile (e.g. Xilouris et al. 1998). PSR B0656+14 is $92 \%$ linear polarized at $400 \mathrm{MHz}$ and also the position angle is remarkably flat (Gould \& Lyne 1998). An interesting comparison might be drawn between the B0656+14 and a number of millisecond pulsars which are also known to have slowly-varying unstable profiles (e.g. Ramachandran \& Kramer 2003; Kramer et al. 1999b; Backer \& Sallmen 1997), possibly resulting from spiky emission in one or more components.

\subsection{The spiky emission}

We show in this paper that the emission of PSR B0656+14 can be characterized by spiky emission that is not confined to a narrow pulse-longitude range, but generally concentrated toward the centre of its profile. This spiky emission has a low occurrence rate within each pulse and is often quasi-periodic in structure. In addition, pulses with spiky emission have a tendency to cluster in successive pulses. By contrast, there is also a weak emission, which appears with a high occurrence rate over the full width of the pulse and varies little from pulse to pulse. Furthermore, the profiles of the spiky and weak emission have very different shapes and frequency evolution. Taken together, these properties all argue for two distinct types of emission.

Separate properties of weak and strong emission can be supported by the fits of the pulse-energy distribution. However, in Fig. 10 one can see that all pulses with a peak-flux weaker than the average are below the noise level. Therefore more sensitive observations (with at least an order of magnitude increase in $S / N$ ) are required to see single weak pulses and to find out if there exists a break in the pulse-energy distribution. Such an observation would show if the spiky and weak emission are associated with the extreme ends of a single smooth energy distribution, or if they are two physically distinct distributions.

Pulse profiles of many pulsars can accurately be decomposed into a small number of Gaussian emission components (Kramer et al. 1994). This is not possible for the isosceles shape of the profile of the spiky emission at high frequencies. This, together with the width of the central component that is invariant with frequency, may suggest that it reflects a permanent geometrical feature. It might be related to an intensity-enhancing (e.g. caustic) effect that boosts the otherwise weak emission. Due to the low occurence rate of the spiky emission, the profile requires an unusually long timescale to achieve stability (over 25000 pulses at $327 \mathrm{MHz}$ ). Despite the high variability in shape of the spiky emission profile on short timescales, we found that the radio power output remains remarkably stable and this may indicate

\footnotetext{
2 However, it should be noted that invariant profile widths is not a property confined to millisecond pulsars. (Mitra \& Rankin 2002) have shown that a whole class of bright slow pulsars have component widths and component separations independent of frequency.
} 
that processes "repackage" the power within the magnetosphere, rather than generate it.

The emission of PSR B0656+14 shows a quasi-periodicity of $20 P_{1}$, which is intensity modulated, rather than phase modulated. Similar fluctuations without drift are often associated with core emission within a conal structure (e.g. PSR B1237+25, Srostlik \& Rankin 2005). To the best of our knowledge, there have been no theoretical attempts to account for the periodicity of this emission. Possibly the modulation should be considered in the framework of the charge flow balance within the entire magnetosphere. Although we conventionally suppose in such a pulsar that we only have a view of one pole, it has recently been plausibly suggested (Dyks et al. 2005) that in selected stars (all of them pulsed X-ray sources, as is PSR B0656+14) it is possible to clearly detect radiation from particles downflowing towards the pole that is hidden from our view. The widely-separated high-energy peaks located well away from the phase of the polar cap point to activity in the outer magnetosphere, possibly associated with an outer gap. Also in the case of PSR B0656+14 we may be dealing with emission which is stimulated, either directly on indirectly, by an inflow - as well as an outflow - of charged particles (an analogy with rainfall is not inappropriate), with short-lived showers of particles intermittently injected into the polar regions in a quasi-periodic manner. This idea will be explored in a subsequent paper, in which we will also present polarization data and attempt to link the radio emission to the high-energy peaks.

Acknowledgements. G.A.E.W. and J.M.R. thank the Netherlands Foundation for Scientific Research (NWO) and the Anton Pannekoek Institute, Amsterdam, for their kind hospitality, and GAEW the University of Sussex for a Visiting Fellowship. Portions of this work (for JMR) were carried out with support from US National Science Foundation Grants AST 99-87654 and 00-98685. Arecibo Observatory is operated by Cornell University under contract to the US National Science Foundation. The Westerbork Synthesis Radio Telescope is operated by the ASTRON (Netherlands Foundation for Research in Astronomy) with support from NWO.

\section{References}

Backer, D. C. 1970, Nature, 227, 692

Backer, D. C., \& Sallmen, S. 1997, AJ, 114, 1539

Backus, P. R. 1981, Ph.D. Thesis, The University of Massachusetts

Becker, W., \& Trü, J. 1997, A\&A, 326, 682
Bhat, N. D. R., Cordes, J. M., Camilo, F., Nice, D. J., \& Lorimer, D. R. 2004, ApJ, 605, 759

Cairns, I. H. 2004, ApJ, 610, 948

Cairns, I. H., Johnston, S., \& Das, P. 2001, ApJ, 563, L65

Cheng, K. S., \& Zhang, L. 1999, ApJ, 515, 337

Cognard, I., Shrauner, J. A., Taylor, J. H., \& Thorsett, S. E. 1996, ApJ, 457, 81 Cordes, J. M. 1978, ApJ, 222, 1006

De Luca, A., Caraveo, P. A., Mereghetti, S., Negroni, M., \& Bignami, G. F. 2005, ApJ, 623, 1051

Dyks, J., Fra̧ckowiak, M., Słowikowska, A., Rudak, B., \& Zhang, B. 2005, ApJ, 663, 1101

Edwards, R. T., \& Stappers, B. W. 2002, A\&A, 393, 733

Edwards, R. T., \& Stappers, B. W. 2003, A\&A, 407, 273

Gould, D. M., \& Lyne, A. G. 1998, MNRAS, 301, 235

Hankins, T. H., Kern, J. S., Weatherall, J. C., \& Eilek, J. A. 2003, Nature, 422, 141

Harding, A. K., \& Muslimov, A. G. 1998, ApJ, 500, 862

Helfand, D. J., Manchester, R. N., \& Taylor, J. H. 1975, ApJ, 198, 661

Johnston, S., \& Romani, R. 2002, MNRAS, 332, 109

Johnston, S., van Straten, W., Kramer, M., \& Bailes, M. 2001, ApJ, 549, L101

Kern, B., Martin, C., Mazin, B., \& Halpern, J. P. 2003, ApJ, 597, 1049

Kinkhabwala, A., \& Thorsett, S. E. 2000, ApJ, 535, 365

Kramer, M., Wielebinski, R., Jessner, A., Gil, J. A., \& Seiradakis, J. H. 1994, A\&AS, 107, 515

Kramer, M., Lange, C., Lorimer, D. R., et al. 1999a, ApJ, 526, 957

Kramer, M., Xilouris, K. M., Camilo, F., et al. 1999b, ApJ, 520, 324

Kramer, M., Karastergiou, A., Gupta, Y., et al. 2003, A\&A, 407, 655

Kuzmin, A. D., \& Ershov, A. A. 2004, A\&A, 427, 575

Lundgren, S. C., Cordes, J. M., Ulmer, M., et al. 1995, ApJ, 453, 433

Mitra, D., \& Rankin, J. M. 2002, ApJ, 322

Pavlov, G. G., Zavlin, V. E., \& Sanwal, D. 2002, in Proceedings of the 270-th WE-Heraeus Seminar, Neutron Stars, Pulsars and Supernova Remnants, ed. W. Becker, H. Lesch, \& J. Truemper [arXiv: astro-ph/0206024]

Press, W. H., Flannery, B. P., Teukolsky, S. A., \& Vetterling, W. T. 1986, Numerical Recipes: The Art of Scientific Computing (Cambridge: Cambridge University Press)

Ramachandran, R., \& Kramer, M. 2003, A\&A, 407, 1085

Rathnasree, N., \& Rankin, J. M. 1995, ApJ, 452, 814

Romani, R., \& Johnston, S. 2001, ApJ, 557, L93

Scheuer, P. A. G. 1968, Nature, 218, 920

Shibanov, Y. A., Sollerman, J., Lundqvist, P., Gull, T., \& Lindler, D. 2005, A\&A, 440,693

Soglasnov, V. A., Popov, M. V., Bartel, N., et al. 2004, ApJ, 616, 439

Srostlik, Z., \& Rankin, J. M. 2005, MNRAS, 362, 1121

Staelin, D. H., \& Reifenstein, E. C. 1968, Science, 162, 1481

Weltevrede, P., Edwards, R. T., \& Stappers, B. W. 2006a, A\&A, 445, 243

Weltevrede, P., Stappers, B. W., Rankin, J. M., \& Wright, G. A. E. 2006b, ApJ, 645, L149

Xilouris, K. M., Kramer, M., Jessner, A., et al. 1998, ApJ, 501, 286

Zavlin, V. E., Pavlov, G. G., \& Shibanov, Y. A. 1996, A\&A, 315, 141

Zhang, B., Sanwal, D., \& Pavlov, G. G. 2005, ApJ, 624, L109 